NBER WORKING PAPER SERIES

\title{
A PREFERRED-HABITAT MODEL OF THE TERM STRUCTURE OF INTEREST RATES
}

\author{
Dimitri Vayanos \\ Jean-Luc Vila \\ Working Paper 15487 \\ http://www.nber.org/papers/w15487
NATIONAL BUREAU OF ECONOMIC RESEARCH
1050 Massachusetts Avenue
Cambridge, MA 02138 \\ November 2009
}

We thank Markus Brunnermeier, Andrea Buraschi, Pierre Collin-Dufresne, Peter DeMarzo, Giorgio Fossi, Ken Garbade, Robin Greenwood, Moyeen Islam, Arvind Krishnamurthy, Jun Liu, Vasant Naik, Anna Pavlova, Jeremy Stein, seminar participants at the Bank of England, Chicago Fed, ECB, LSE, Manchester, New York Fed, Tilburg, Toulouse, UCLA, and participants at the American Finance Association 2008, Adam Smith Asset Pricing 2007, Brazilian Finance Association 2008, Chicago 2008, CRETE 2008, Gerzensee 2007, Imperial 2007, NBER Asset Pricing 2007, and SITE 2006 conferences for helpful comments. We have especially benefited from an extensive set of insightful comments by John Cochrane, and from a communication by Xavier Gabaix on linearity-generating processes. Financial support from the Paul Woolley Centre at the LSE is gratefully acknowledged. The views expressed in this paper are those of the authors and not of Bank of America Merrill Lynch, any of its affiliates, or the National Bureau of Economic Research.

NBER working papers are circulated for discussion and comment purposes. They have not been peerreviewed or been subject to the review by the NBER Board of Directors that accompanies official NBER publications.

(C) 2009 by Dimitri Vayanos and Jean-Luc Vila. All rights reserved. Short sections of text, not to exceed two paragraphs, may be quoted without explicit permission provided that full credit, including $\odot$ notice, is given to the source. 
A Preferred-Habitat Model of the Term Structure of Interest Rates

Dimitri Vayanos and Jean-Luc Vila

NBER Working Paper No. 15487

November 2009

JEL No. E4,E5,G1

\begin{abstract}
$\underline{\text { ABSTRACT }}$
We model the term structure of interest rates as resulting from the interaction between investor clienteles with preferences for specific maturities and risk-averse arbitrageurs. Because arbitrageurs are risk averse, shocks to clienteles' demand for bonds affect the term structure---and constitute an additional determinant of bond prices to current and expected future short rates. At the same time, because arbitrageurs render the term structure arbitrage-free, demand effects satisfy no-arbitrage restrictions and can be quite different from the underlying shocks. We show that the preferred-habitat view of the term structure generates a rich set of implications for bond risk premia, the effects of demand shocks and of shocks to short-rate expectations, the economic role of carry trades, and the transmission of monetary policy.
\end{abstract}

Dimitri Vayanos

Department of Finance, A350

London School of Economics

Houghton Street

London WC2A 2AE

UNITED KINGDOM

and CEPR

and also NBER

d.vayanos@1se.ac.uk

Jean-Luc Vila

Bank of America Merrill Lynch

2 King Edward Street

London EC1A 1HQ

United Kingdom

Jean-Luc.Vila@baml.com 


\section{Introduction}

What determines the interest rate for a given maturity? Standard economic theory links the interest rate for a maturity $T$ to the willingness of a representative agent to substitute consumption between times 0 and $T$. This model of interest-rate determination contrasts sharply with a more informal preferred-habitat view, proposed by Culbertson (1957) and Modigliani and Sutch (1966), and popular among practitioners. According to that view, there are investor clienteles with preferences for specific maturities, and the interest rate for a given maturity is influenced by demand and supply shocks local to that maturity. ${ }^{1}$

The preferred-habitat view is supported by numerous market episodes. One example is the 2000-2002 buyback program by the US Treasury. In January 2000, the Treasury announced its intention to buy back long-term bonds through a series of reverse auctions. ${ }^{2}$ Within three weeks of this announcement, yields on thirty-year bonds had dropped by 58bps (hundredths of one percent), a price increase larger than ten percent. Moreover, the effect was confined to long maturities: yields on five-year bonds dropped by only 9bps, while yields on two-year bonds rose by 9bps. These effects are hard to rationalize within a representative-agent model: one would have to argue that the buyback program signalled a significant drop in aggregate consumption in thirty years. On the other hand, the effects are consistent with the preferred-habitat view since the buyback program was a supply shock local to long maturities.

Even though the preferred-habitat view is relevant in practice and has been proposed more than half a century ago, it has not entered into the academic mainstream; it has typically been confined to a short discussion in MBA-level textbooks. This might be partly because of the absence of a formal model, and partly because of an impression that preferred habitat can conflict with the logic of no-arbitrage. Indeed, an extreme version of the preferred-habitat view is that the interest rate for a given maturity evolves independently of nearby maturities. This conflicts with no-arbitrage, as pointed out by Cox, Ingersoll and Ross (1985).

In this paper, we build a formal model of preferred habitat. We assume that the term structure

\footnotetext{
${ }^{1}$ The typical clientele for bonds with maturities longer than fifteen years are pension funds, seeking to hedge their long-term pension liabilities. Life-insurance companies are typically located around the fifteen-year mark, while asset managers and banks' treasury departments are the typical clientele for bonds with maturities shorter than ten years.

${ }^{2}$ The objective of the buyback program was to shorten the average maturity of government debt, which had increased during the late 1990s because of the budget surpluses. (Expiring bond issues were not being replaced, and most of them were short term.) The buyback program was conducted through 45 reverse auctions between March 2000 and April 2002. The maturities of the targeted issues ranged from 10 to 27 years, and an average of $14 \%$ of each targeted issue was bought back. For accounts of this episode, see Garbade and Rutherford (2007) and Greenwood and Vayanos (2009b). The latter paper discusses additional episodes supporting the preferred-habitat view: the 2004 pension reform in the UK and the 2008-2009 quantitative easing by the Federal Reserve.
} 
of interest rates is determined through the interaction between investor clienteles with preferences for specific maturities and risk-averse arbitrageurs. Arbitrageurs integrate maturity markets, ensuring that bonds with nearby maturities trade at similar prices and the term structure is arbitrage-free. But because arbitrageurs are risk averse, the demand shocks of clienteles affect the term structure. Understanding how demand effects manifest themselves in the cross-section of maturities, and in a way consistent with no-arbitrage, is central to our analysis. We show that demand effects can be quite different from the underlying shocks, in ways that depend on arbitrageur risk aversion and the underlying risk factors. Besides addressing the effects of demand shocks, our model generates a rich set of implications for bond risk premia, the effects of shocks to short-rate expectations, the economic role of carry trades, and the transmission of monetary policy.

Our model, described in Section 2, is set in continuous time. The short rate follows an exogenous mean-reverting process, and interest rates for longer maturities are determined endogenously through trading between investors and arbitrageurs. ${ }^{3}$ Investors constitute clienteles with preferences for specific maturities. We assume that preferences take an extreme form, whereby the clientele for a given maturity demands only the zero-coupon bond with the same maturity. ${ }^{4}$ Thus, in the absence of arbitrageurs, the term structure would exhibit extreme segmentation: the interest rate for a given maturity would be influenced only by the demand of the corresponding clientele, and would evolve independently of other maturities. Of course, such segmentation does not occur in equilibrium because of arbitrageurs. We assume that arbitrageurs can invest in all maturities, and maximize a mean-variance objective over instantaneous changes in wealth. Our assumptions on investors and arbitrageurs ensure that the model has a tractable structure, with equilibrium interest rates being affine in the state variables.

Section 3 considers the case where clientele demands are constant over time. In the absence of arbitrageurs, the term structure would also be constant, and disconnected from the time-varying short rate. Arbitrageurs bridge this disconnect, incorporating information about current and future short rates into bond prices. Suppose, for example, that the short rate increases, thus becoming attractive relative to investing in bonds. Because investors do not venture away from their maturity habitats, they do not take advantage of this opportunity. Arbitrageurs, however, do take advantage by shorting bonds and investing at the short rate. Through this carry ("roll-up") trade, bond prices drop and yields rise. ${ }^{5}$ Conversely, a negative shock that lowers the short rate induces arbitrageurs

\footnotetext{
${ }^{3}$ The short rate concerns borrowing and lending over an infinitesimal maturity. The assumption of an exogenous short rate is common in the literature, e.g., Vasicek (1977). See also Cox, Ingersoll and Ross (1985), who derive the short rate from the exogenous return of a risky technology producing output over an infinitesimal maturity.

${ }^{4}$ We tie clientele demands to optimizing behavior in Appendix B, in a setting where overlapping generations of investors consume at the end of their life and are infinitely risk averse.

${ }^{5}$ Carry trades are trades that are profitable if the market environment does not change. For example, shorting
} 
to borrow at the short rate and buy bonds. Through this carry ("roll-down") trade, bond prices rise and yields drop. In both cases, the carry trades of arbitrageurs provide the mechanism through which bond yields move to reflect changes in current and expected future short rates.

Since carry trades are risky, they must offer positive expected returns to attract risk-averse arbitrageurs. This has implications for bond risk premia. When the short rate is high, arbitrageurs short bonds and invest at the short rate. Therefore, bonds earn negative premia, offering lower expected returns than the short rate. Conversely, premia are positive when the short rate is low because arbitrageurs borrow at the short rate and buy bonds. Our model thus offers an explanation for the puzzling finding of Fama and Bliss (1987) that bond risk premia switch sign from positive during times when the term structure slopes up to negative when it slopes down.

Our analysis has implications for the transmission of monetary policy. Suppose that the Central Bank conducts monetary policy through the short rate. Since the effects of this policy are propagated along the term structure through the carry trades of arbitrageurs, the extent of propagation depends on arbitrageur risk aversion. When, for example, risk aversion is high (e.g., arbitrageurs are under-capitalized), propagation is limited and forward rates under-react severely to changes in expected short rates. Note that in acting as conduits of monetary policy, arbitrageurs reap rewards in the form of positive expected returns from their carry trades. Thus, monetary policy transfers wealth to arbitrageurs in expected terms. For example, a policy that keeps the short rate low transfers wealth to arbitrageurs through positive bond risk premia. ${ }^{6}$

Section 4 considers the case where clientele demands are driven by one common mean-reverting factor. Bond yields are then driven by two risk factors: short rate and demand. We allow the demand factor to take a general form, e.g., it can be a local shock that impacts only the clientele for a specific maturity, or a global shock that impacts all clienteles. One would expect that the price effects of local shocks are not fully local because they are transmitted to nearby maturities by arbitrageurs. But to what extent is transmission effective? And more broadly, how does the location of demand shocks in the maturity space relate to their effect on the term structure?

When arbitrageur risk aversion is low, the location of demand shocks matters only for the overall magnitude of their effect but not for the effect's relative importance across maturities. Compare, for example, a decrease in demand of the twenty-year clientele to the same shock to the five-year clientele. The twenty-year shock has a larger effect on the term structure because arbitrageurs

bonds and investing at the short rate is profitable if the short rate remains high. This trade is commonly referred to as roll-up because it is a bet that the short rate will remain high, in which case bond yields will roll up to that high value as bonds mature.

${ }^{6}$ We are grateful to John Cochrane for suggesting this idea in his discussion of our work (Cochrane (2008)). 
must be induced to buy twenty-year bonds, which are more sensitive to short-rate risk. Yet, both shocks have the same relative effect across maturities: if, for example, the twenty-year shock has its largest effect on the fifteen-year yield, the same is true for the five-year shock. The intuition is that when arbitrageur risk aversion is low, the short rate is the dominant risk factor, and demand shocks affect the term structure by altering the market price of short-rate risk. Bonds most heavily affected by the shocks are those most sensitive to changes in the market price of short-rate risk, a characteristic which is independent of the shocks' location.

Our model refines and qualifies the logic of limited arbitrage. Consistent with that logic, we find that demand shocks affect prices because arbitrageurs' risk tolerance is limited. Yet, even with limited risk tolerance, arbitrageurs are able to eliminate riskfree arbitrage opportunities. As a consequence, arbitrage - even limited - imposes tight restrictions on how demand shocks affect the cross-section of bonds: the effects must be through the factor prices of risk. When arbitrageur risk aversion is low, the restrictions are particularly tight because the short rate is effectively the only risk factor. When instead arbitrageurs are concerned with multiple risk factors, the restrictions become looser, and demand effects acquire a preferred-habitat flavor. Indeed, when arbitrageur risk aversion is high, and so arbitrageurs are concerned with both the short-rate and the demand factor, demand shocks located at longer maturities generate effects that are largest at longer maturities. Moreover, when additional demand factors are introduced, in an extension sketched in Section 5, the effects of local demand shocks tend to become more local.

Our emphasis in this paper is mainly qualitative: use the closed-form solutions to understand key intuitions and comparative statics. We conclude this paper in Section 6 by arguing that an equally rich set of implications could be derived on the quantitative front. In particular, structural estimation of the model could render it a valuable policy tool for addressing questions such as how purchases of bonds by the Central Bank, or issuance by the Treasury, can affect the term structure. Our model is uniquely able to address such questions because of its focus on demand and supply shocks.

A number of recent papers seek to explain the positive relationship between bond risk premia and the slope of the term structure. In Wachter (2006), Buraschi and Jiltsov (2007) and Lettau and Wachter (2009), a representative agent has habit formation, and periods of low consumption are associated with high short rates and bond risk premia. The time-variation in premia generates a positive relationship with slope, but in contrast to our model premia are always positive. In Gabaix (2009), time-variation in premia arises because of the time-varying severity of rare disasters facing a representative agent. In Xiong and Yan (2009), two agents hold heterogeneous beliefs about the 
time-varying mean of the short rate. When agents are overly optimistic about the mean, they undervalue the bonds, and this leads an econometrician who infers the mean correctly to observe positive premia and positive slope. While we do not dispute the relevance of habit formation, rare disasters, and heterogeneous beliefs for asset pricing, we believe that in episodes such as the Treasury buyback, changes in risk premia were generated by an entirely different mechanism. Considering this mechanism yields a new set of intuitions and predictions.

A recent literature explores the empirical implications of preferred habitat, as well as the implications for bond issuance. Krishnamurthy and Vissing-Jorgensen (2008) find a strong negative correlation between credit spreads and the debt-to-gdp ratio, and argue that this reflects a downward-sloping demand for government bonds. Greenwood and Vayanos (2009a) find that the average maturity of government debt predicts positively excess bond returns, a result they also derive theoretically in an extension of our model. Guibaud, Nosbusch and Vayanos (2009) show that catering to maturity clienteles is an optimal issuance policy: a welfare-maximizing government issues more long-term debt when the fraction of long- relative to short-horizon investors increases. Greenwood, Hanson and Stein (2009) find that corporations engage in gap-filling behavior, issuing long-term debt at times when the supply of long-term government debt is small. ${ }^{7}$

Finally, our work is related to papers studying the pricing of multiple assets within a class when arbitrage is limited. In Barberis and Shleifer (2003), arbitrageurs absorb demand shocks of investors with preferences for specific asset styles. These shocks generate comovement of assets within a style. In Pavlova and Rigobon (2008), style arises because of portfolio constraints, and can be the source of international financial contagion. ${ }^{8}$ In Greenwood (2005), risk-averse arbitrageurs absorb demand shocks of index investors. A demand shock for one stock affects other stocks through the covariance with the arbitrageurs' portfolio, a property confirmed empirically using data on index redefinitions. ${ }^{9}$ In Gabaix, Krishnamurthy and Vigneron (2007), the marginal holders of mortgage-backed securities are risk-averse arbitrageurs, whose wealth is tied to a mortgage portfolio rather than to economywide wealth. Consistent with this idea, pre-payment risk is found to be priced according to the covariance with the mortgage portfolio. In Garleanu, Pedersen and Poteshman (2009), the marginal traders in the options market are risk-averse market makers who absorb demand shocks of other investors. This yields implications for how demand shocks should affect the cross-section of options, which are confirmed empirically using measures of demand pressure.

\footnotetext{
${ }^{7}$ See also Bakshi and Chen (1996) who derive preferred habitats from trading frictions, and Telmer and Zin (1996) who link preferred habitats to investor portfolio holdings.

${ }^{8}$ Kyle and Xiong (2001) derive contagion from the wealth effects of arbitrageurs with logarithmic preferences, and Gromb and Vayanos $(2002,2009)$ derive contagion from arbitrageurs' margin constraints.

${ }^{9} \mathrm{Hau}(2009)$ extends this analysis by introducing unsophisticated liquidity suppliers alongside the arbitrageurs.
} 


\section{Model}

Time is continuous and goes from zero to infinity. The term structure at time $t$ consists of a continuum of zero-coupon bonds in zero supply. The maturities of the bonds are in the interval $(0, T]$, and the bond with maturity $\tau$ pays $\$ 1$ at time $t+\tau$. We denote by $P_{t, \tau}$ the time- $t$ price of the bond with maturity $\tau$, by $R_{t, \tau}$ the spot rate for that maturity, and by $f_{t, \tau-\Delta \tau, \tau}$ the forward rate between maturities $\tau-\Delta \tau$ and $\tau$. The spot rate is related to the price through

$$
R_{t, \tau}=-\frac{\log \left(P_{t, \tau}\right)}{\tau}
$$

and the forward rate through

$$
f_{t, \tau-\Delta \tau, \tau}=-\frac{\log \left(\frac{P_{t, \tau}}{P_{t, \tau-\Delta \tau}}\right)}{\Delta \tau} .
$$

The short rate $r_{t}$ is the limit of $R_{t, \tau}$ when $\tau$ goes to zero. We take $r_{t}$ as exogenous and assume that it follows the Ornstein-Uhlenbeck process

$$
d r_{t}=\kappa_{r}\left(\bar{r}-r_{t}\right) d t+\sigma_{r} d B_{r, t}
$$

where $\left(\bar{r}, \kappa_{r}, \sigma_{r}\right)$ are positive constants and $B_{r, t}$ is a Brownian motion. The short rate $r_{t}$ could be determined by the Central Bank and the macro-economic environment, but we do not model these mechanisms. Our focus instead is on how exogenous movements in $r_{t}$ influence the bond prices $P_{\tau, t}$ that are endogenously determined in equilibrium.

Agents are of two types: preferred-habitat investors and arbitrageurs. Preferred-habitat investors constitute maturity clienteles, with the clientele for maturity $\tau$ demanding the bond with the same maturity. We assume that the demand for the bond with maturity $\tau$, expressed in time $t$ dollars, is an increasing and linear function of the bond's yield $R_{t, \tau}$, i.e.,

$$
y_{t, \tau}=\alpha(\tau) \tau\left(R_{t, \tau}-\beta_{t, \tau}\right)
$$

We impose no restrictions on the function $\alpha(\tau)$ except that it is positive.

If preferred-habitat investors were the only market participants, then the term structure would exhibit extreme segmentation: the yield for a given maturity would be influenced only by the demand of the corresponding clientele, and would evolve independently of other maturities. Given the demand (4) and bonds' zero supply, the equilibrium yield for maturity $\tau$ would be $R_{t, \tau}=\beta_{t, \tau}$. 
Of course, such segmentation does not occur in equilibrium because of arbitrageurs. Arbitrageurs integrate maturity markets, ensuring that bonds with nearby maturities trade at similar prices and the term structure is arbitrage-free.

In Appendix B we show that optimizing behavior yields the demand (4) with the slight difference that (4) concerns units of the bond rather than time $t$ dollars. ${ }^{10}$ We assume that there are overlapping generations of investors, who consume at the end of their life and are infinitely risk averse. Infinite risk aversion ensures that investors demand only the bond that matures at the end of their life. ${ }^{11}$ Therefore, each generation constitutes a clientele for a specific maturity. We additionally assume that investors can save for consumption either through bonds or through a private technology that is an imperfect substitute. This ensures that demand is elastic in the yield $R_{t, \tau}$, with $\beta_{t, \tau}$ being the return on the private technology.

Assuming that preferred-habitat investors demand only the bond corresponding to their maturity habitat is, of course, extreme. This assumption, however, renders the analysis manageable, while not detracting from our main focus which is how limited arbitrage can integrate segmented maturity markets. Indeed, if preferred-habitat investors could move away from their maturity habitat, they would do so when other bonds offer more attractive returns. Therefore, they would contribute to the integration of markets, which in our model is done solely by arbitrageurs. This would enlarge the set of arbitrageurs without affecting the qualitative features of our analysis. At the same time, the analysis would become more complicated because the portfolio of each maturity clientele would consist of a continuum of bonds rather than a single bond.

While we preclude preferred-habitat investors from substituting across maturities, we allow them to substitute outside the bond market through the private technology. This type of substitution is important for some aspects of our analysis. At a fundamental level, our analysis centers around how limited arbitrage can integrate segmented maturity markets. These segmented markets can clear (at the yield $\beta_{t, \tau}$ ) only if the demand of preferred-habitat investors is elastic in the yield of the bond corresponding to their maturity habitat. Allowing for substitution outside the bond

\footnotetext{
${ }^{10}$ Since the demand expressed in dollars is derived from that in units by multiplying by the bond price, the dollar demand derived in Appendix B has the form (4) but with $\alpha(\tau)$ depending on $t$. We assume that the demand (4) concerns dollars rather than units because when combining with an Ornstein-Uhlenbeck short-rate process, we find equilibrium spot rates that are affine in the state variables. In Appendix B we present a modification of our model where optimizing behavior by preferred-habitat investors yields a demand expressed in units that has the form (4) except that it is linear and decreasing in the bond price rather than linear and increasing in the bond yield. When combining with a short-rate process that belongs to the class of Gabaix's (2009) linearity-generating processes, we find equilibrium bond prices that are affine in the state variables.

${ }^{11}$ More precisely, investors demand a riskless payoff at the end of their life. They can achieve this payoff by holding either the bond that matures at the end of their life or a replicating portfolio. If the term structure is arbitrage-free, as is the case in equilibrium, the replicating portfolio costs the same as the bond. Therefore, investors are indifferent between the two, and we assume that they hold the bond.
} 
market generates this elasticity. In later sections we show that elastic demand underlies some of our main results, e.g., the effects of local demand shocks can be tied to the shocks' location, and bond risk premia switch sign according to the slope of the term structure. ${ }^{12} 13$

We assume that the intercept $\beta_{t, \tau}$ in the demand (4) takes the form

$$
\beta_{t, \tau}=\bar{\beta}+\sum_{k=1}^{K} \theta_{k}(\tau) \beta_{k, t}
$$

where $\bar{\beta}$ is a constant, $\left\{\beta_{k, t}\right\}_{k=1, . ., K}$ are demand risk factors and $\left\{\theta_{k}(\tau)\right\}_{k=1, . ., K}$ are functions characterizing how each factor would impact the cross-section of maturities in the absence of arbitrageurs. For example, when $\theta_{k}(\tau)$ is independent of $\tau$, a change in $\beta_{k, t}$ would impact all maturities equally and cause a parallel shift in the term structure. When instead $\theta_{k}(\tau)$ is single-peaked around a specific maturity, a change in $\beta_{k, t}$ would impact that maturity the most, and can be interpreted as a local demand shock. We impose no restrictions on the functions $\left\{\theta_{k}(\tau)\right\}_{k=1, \ldots, K}$.

The demand factors can be given a number of interpretations. In Appendix B we interpret them as returns on investments outside the bond market, e.g., real estate. Demand factors could alternatively be interpreted as changes in the hedging needs of preferred-habitat investors (arising because of, e.g., changes in pension funds' liabilities or regulation), changes in the size or composition of the preferred-habitat investor pool, or changes in the supply of bonds issued by the government. We assume that the demand factor $\beta_{k, t}$ follows the Ornstein-Uhlenbeck process

$$
d \beta_{k, t}=-\kappa_{\beta, k} \beta_{k, t} d t+\sigma_{\beta, k} d B_{\beta, k, t},
$$

where $\left(\kappa_{\beta, k}, \sigma_{\beta, k}\right)$ are positive constants and $B_{\beta, k, t}$ is a Brownian motion independent of $B_{r, t}$ and $B_{\beta, k^{\prime}, t}$ for $k^{\prime} \neq k^{14}$

\footnotetext{
${ }^{12}$ Footnotes 15 and 23 explain why these results follow from elastic demand.

${ }^{13}$ Substitution by preferred-habitat investors towards non-bond investments is relevant in practice. Consider, for example, the 2004 pension reform in the UK, which required pension funds to evaluate their long-term pension liabilities using market long rates. To hedge against changes in long rates, pension funds tilted their portfolios towards long-term bonds, and this drove long rates to record low levels. The drop in long rates induced pension funds to substitute towards both shorter-maturity bonds and non-bond investments. The non-bond investments included real estate: for example, Marks \& Spencer arranged for their pension fund to receive payments based on the leases of their property portfolio. For accounts of the UK pension reform and its effect on the term structure, see the Barclays Capital reports by Tzucker and Islam (2005) and Islam (2007), as well as Greenwood and Vayanos (2009b). The Marks \& Spencer deal is mentioned in Islam (2007), p.61. Tzucker and Islam (2005), p.10, emphasize the elasticity of pension-fund demand for long-term bonds:

... The market experience has been that pension funds and other "real money" investors will be buyers of real yields on an outright basis when yields are higher than 1.50-1.60\%. It is salutary to note that this "bid-only" level was $2.20-2.40 \%$ in the earlier half of this decade...

In our model, the buy threshold corresponds to the intercept $\beta_{t, \tau}$ in the demand (4). Note that this threshold is time-varying, and changed because of the pension reform.

${ }^{14}$ Allowing demand factors to be correlated with each other and with the short rate complicates the formulas without affecting the main results. See the discussion at the end of Section 4.
} 
Arbitrageurs choose a bond portfolio to trade off instantaneous mean and variance. Denoting their time- $t$ wealth by $W_{t}$ and their dollar investment in the bond with maturity $\tau$ by $x_{t, \tau}$, their budget constraint is

$$
d W_{t}=\left(W_{t}-\int_{0}^{T} x_{t, \tau}\right) r_{t} d t+\int_{0}^{T} x_{t, \tau} \frac{d P_{t, \tau}}{P_{t, \tau}} .
$$

The arbitrageurs' optimization problem is

$$
\max _{\left\{x_{t, \tau}\right\}_{\tau \in(0, T]}}\left[E_{t}\left(d W_{t}\right)-\frac{a}{2} \operatorname{Var}_{t}\left(d W_{t}\right)\right]
$$

where $a$ is a risk-aversion coefficient, characterizing the trade-off between mean and variance.

Because arbitrageurs render the term structure arbitrage-free, they eliminate riskfree arbitrage opportunities. Therefore, their positions in equilibrium involve risk, and their returns derive from the premium associated to that risk rather than from riskfree arbitrage opportunities. This describes most of term-structure arbitrage, done by, e.g., hedge funds and proprietary-trading desks.

We endow arbitrageurs with preferences over instantaneous mean and variance for analytical convenience. One interpretation of these preferences is that there are overlapping generations of arbitrageurs, each living for an infinitesimal time interval. Intertemporal optimization of long-lived arbitrageurs with logarithmic utility would also give rise to preferences over instantaneous mean and variance, but the risk-aversion coefficient $a$ would depend on wealth. In taking $a$ to be constant, we suppress wealth effects. We appeal informally to wealth effects, however, when drawing some of the model's empirical implications.

\section{One-Factor Model}

This section studies the case where there are no demand factors $(K=0)$. The only risk factor is the short rate $r_{t}$, and the term structure is described by a one-factor model. The one-factor model yields some of our main results, while being analytically very simple.

\subsection{Equilibrium}

We conjecture that equilibrium spot rates are affine in $r_{t}$, i.e.,

$$
P_{t, \tau}=e^{-\left[A_{r}(\tau) r_{t}+C(\tau)\right]}
$$


for two functions $A_{r}(\tau), C(\tau)$ that depend on maturity $\tau$. Applying Ito's Lemma to $(9)$ and using the dynamics (3) of $r_{t}$, we find that the instantaneous return on the bond with maturity $\tau$ is

$$
\frac{d P_{t, \tau}}{P_{t, \tau}}=\mu_{t, \tau} d t-A_{r}(\tau) \sigma_{r} d B_{r, t}
$$

where

$$
\mu_{t, \tau} \equiv A_{r}^{\prime}(\tau) r_{t}+C^{\prime}(\tau)-A_{r}(\tau) \kappa_{r}\left(\bar{r}-r_{t}\right)+\frac{1}{2} A_{r}(\tau)^{2} \sigma_{r}^{2}
$$

is the instantaneous expected return. Substituting (10) into the arbitrageurs' budget constraint (7), we can solve the arbitrageurs' optimization problem (8).

Lemma 1. The arbitrageurs' first-order condition is

$$
\mu_{t, \tau}-r_{t}=A_{r}(\tau) \lambda_{r, t}
$$

where

$$
\lambda_{r, t} \equiv a \sigma_{r}^{2} \int_{0}^{T} x_{t, \tau} A_{r}(\tau) d \tau
$$

Eq. (12) requires that a bond's expected excess return $\mu_{t, \tau}-r_{t}$ is proportional to the bond's sensitivity $A_{r}(\tau)$ to the short rate. The proportionality coefficient $\lambda_{r, t}$ (which is the same for all bonds) is the market price of short-rate risk. Eq. (12) follows solely from the absence of arbitrage, without imposing any of the additional structure of our model. Indeed, since the short rate is the only risk factor, a bond's risk is fully characterized by the sensitivity to the short rate. Absence of arbitrage requires that expected excess return per unit of risk is the same for all bonds. The common value of this ratio is the market price of short-rate risk $\lambda_{r, t}$.

While absence of arbitrage requires that risk is priced consistently across bonds, it imposes essentially no restrictions on the common price of risk $\lambda_{r, t}$. We determine $\lambda_{r, t}$ using the additional structure of our model, namely, the specification of preferred-habitat investors and arbitrageurs. The main economic insights coming out of our model can be traced to properties of $\lambda_{r, t}$ (and of the market price of demand risk in later sections).

Since (12) is the arbitrageurs' first-order condition, $\lambda_{r, t}$ is the expected excess return that arbitrageurs require as compensation for taking a marginal unit of risk. This yields the characterization 
in (13). In particular, arbitrageurs require high compensation for adding risk to their portfolio if the portfolio is highly sensitive to risk, i.e., $\int_{0}^{T} x_{t, \tau} A_{r}(\tau) d \tau$ is high. Moreover, this effect is stronger if arbitrageurs are more risk averse ( $a$ large) or the short rate is more volatile ( $\sigma_{r}^{2}$ large).

The portfolio of arbitrageurs is determined endogenously in equilibrium. Since bonds are in zero supply, arbitrageurs' positions $x_{t, \tau}$ are opposite to the positions $y_{t, \tau}$ of preferred-habitat investors. Setting $x_{t, \tau}=-y_{t, \tau}$ in (13), we can write the market price of short-rate risk $\lambda_{r, t}$ as

$$
\lambda_{r, t}=-a \sigma_{r}^{2} \int_{0}^{T} y_{t, \tau} A_{r}(\tau) d \tau=a \sigma_{r}^{2} \int_{0}^{T} \alpha(\tau)\left[\bar{\beta} \tau-\left[A_{r}(\tau) r_{t}+C(\tau)\right]\right] A_{r}(\tau) d \tau
$$

where the second step follows from (1), (4), (5), (9) and $K=0$. Eq. (14) implies that $\lambda_{r, t}$ is an affine and decreasing function of the short rate $r_{t}$, a property that underlies the main results of this section. Substituting $\mu_{t, \tau}$ from (11) and $\lambda_{r, t}$ from (14) into (12), we find an affine equation in $r_{t}$. Setting linear terms in $r_{t}$ to zero yields

$$
A_{r}^{\prime}(\tau)+\kappa_{r} A_{r}(\tau)-1=-a \sigma_{r}^{2} A_{r}(\tau) \int_{0}^{T} \alpha(\tau) A_{r}(\tau)^{2} d \tau
$$

and setting constant terms to zero yields an equation for $C(\tau)$. Eq. (15) is a linear differential equation in $A_{r}(\tau)$, with the unusual feature that the coefficient of $A_{r}(\tau)$ depends on an integral involving $A_{r}(\tau)$. This is because of a fixed-point problem to which we return later in this section. Proposition 1 reduces the fixed-point problem to a scalar non-linear equation, and determines the functions $A_{r}(\tau), C(\tau)$.

Proposition 1. The functions $A_{r}(\tau), C(\tau)$ are given by

$$
\begin{aligned}
A_{r}(\tau) & =\frac{1-e^{-\kappa_{r}^{*} \tau}}{\kappa_{r}^{*}} \\
C(\tau) & =\kappa_{r}^{*} \bar{r}^{*} \int_{0}^{\tau} A_{r}(u) d u-\frac{\sigma_{r}^{2}}{2} \int_{0}^{\tau} A_{r}(u)^{2} d u,
\end{aligned}
$$

where $\kappa_{r}^{*}$ is the unique solution to

$$
\kappa_{r}^{*}=\kappa_{r}+a \sigma_{r}^{2} \int_{0}^{T} \alpha(\tau) A_{r}(\tau)^{2} d \tau,
$$

and

$$
\bar{r}^{*} \equiv \bar{r}+\frac{(\bar{\beta}-\bar{r}) a \sigma_{r}^{2} \int_{0}^{T} \alpha(\tau) \tau A_{r}(\tau) d \tau+\frac{a \sigma_{r}^{4}}{2} \int_{0}^{T} \alpha(\tau)\left[\int_{0}^{\tau} A_{r}(u)^{2} d u\right] A_{r}(\tau) d \tau}{\kappa_{r}^{*}\left[1+a \sigma_{r}^{2} \int_{0}^{T} \alpha(\tau)\left[\int_{0}^{\tau} A_{r}(u) d u\right] A_{r}(\tau) d \tau\right]} .
$$


The parameters $\left(\bar{r}^{*}, \kappa_{r}^{*}\right)$ characterize the dynamics of the short rate under the risk-neutral measure. In the proof of Proposition 1 we show that these dynamics are

$$
d r_{t}=\kappa_{r}^{*}\left(\bar{r}^{*}-r_{t}\right) d t+\sigma_{r} d \hat{B}_{r, t}
$$

where $\hat{B}_{r, t}$ is a Brownian motion under the risk-neutral measure. The risk-neutral dynamics are thus Ornstein-Uhlenbeck, as are the true dynamics, with long-run mean $\bar{r}^{*}$ and mean-reversion rate $\kappa_{r}^{*}$. Eqs. (16) and (17) are the standard Vasicek (1977) equations, characterizing the term structure when the risk-neutral dynamics are Ornstein-Uhlenbeck.

Identifying how the risk-neutral parameters $\left(\bar{r}^{*}, \kappa_{r}^{*}\right)$ differ from their true counterparts $\left(\bar{r}, \kappa_{r}\right)$ is central to our analysis. The difference between the two sets of parameters is closely related to the properties of the market price of short-rate risk $\lambda_{r, t}$. Risk-neutral and true parameters obviously coincide when arbitrageurs are risk neutral $(a=0)$, as can be seen from (18) and (19).

When arbitrageurs are risk averse, the parameters $\left(\bar{r}^{*}, \kappa_{r}^{*}\right)$ depend on characteristics of the arbitrageurs' portfolio. Since arbitrageurs' positions are opposite to those of preferred-habitat investors, and the latter depend on spot rates, the parameters $\left(\bar{r}^{*}, \kappa_{r}^{*}\right)$ depend on the functions $A_{r}(\tau), C(\tau)$. This gives rise to the fixed-point problem solved in Proposition 1: the risk-neutral parameters $\left(\bar{r}^{*}, \kappa_{r}^{*}\right)$ determine the functions $A_{r}(\tau), C(\tau)$ through the Vasicek equations, but they also depend on these functions.

\subsection{Bond Risk Premia}

In the one-factor model, the term structure in the absence of arbitrageurs is flat at $\bar{\beta}$ and disconnected from the time-varying short rate. Arbitrageurs bridge this disconnect, incorporating information about current and future short rates into bond prices. Examining how arbitrageurs perform this activity reveals key economic mechanisms of our model. It also yields implications for bond risk premia, and ties back to properties of the risk-neutral parameters $\left(\bar{r}^{*}, \kappa_{r}^{*}\right)$ and the market price of short-rate risk $\lambda_{r, t}$.

Suppose that a positive shock raises the short rate above $\bar{\beta}$. This makes investing in the short rate attractive relative to investing in bonds. Because preferred-habitat investors do not venture away from their maturity habitats, they do not take advantage of this opportunity. Arbitrageurs, however, do take advantage by shorting bonds and investing at the short rate. Through this carry ("roll-up") trade, bond prices drop and yields rise. Conversely, a negative shock that lowers the 
short rate induces arbitrageurs to borrow at the short rate and buy bonds. Through this carry ("roll-down") trade, bond prices rise and yields drop. In both cases, the carry trades of arbitrageurs provide the mechanism through which bond yields move to reflect changes in current and expected future short rates.

Bond risk premia reflect arbitrageurs' carry trades. When the short rate is high, arbitrageurs are short bonds through the carry roll-up trade. Since arbitrageurs are the marginal agents, bond risk premia are negative: bonds must offer negative expected returns relative the short rate so that arbitrageurs are induced to short them. Conversely, when the short rate is low, arbitrageurs are long bonds through the carry roll-down trade, and bond risk premia are positive. ${ }^{15}$

Since the short rate is the only source of time-variation in the one-factor model, bond risk premia are positively related to the slope of the term structure: a high (low) short rate implies both a downward (upward) sloping term structure and negative (positive) bond risk premia. The positive slope-premia relationship is consistent with the findings of Fama and Bliss (FB 1987). FB perform the regression

$$
\frac{1}{\Delta \tau} \log \left(\frac{P_{t+\Delta \tau, \tau-\Delta \tau}}{P_{t, \tau}}\right)-R_{t, \Delta \tau}=\alpha_{\mathrm{FB}}+\gamma_{\mathrm{FB}}\left(f_{t, \tau-\Delta \tau, \tau}-R_{t, \Delta \tau}\right)+\epsilon_{t+\Delta \tau}
$$

The dependent variable is the return on a zero-coupon bond with maturity $\tau$ held over a period $\Delta \tau$, in excess of the spot rate for maturity $\Delta \tau$. The independent variable is the forward rate between maturities $\tau-\Delta \tau$ and $\tau$, minus the the spot rate for maturity $\Delta \tau$. FB perform this regression for $\Delta \tau=1$ year and $\tau=2,3,4,5$ years, and find that in all cases $\gamma_{\mathrm{FB}}$ is positive and significant. This means that bond risk premia tend to be positive when forward rates exceed spot rates, i.e., the term structure is upward sloping, and negative when the term structure is downward sloping. The time-variation is significant: the standard deviation of predicted premia is about $1-1.5 \%$ per year, while average premia are about $0.5 \%$ per year. To show the positive slope-premia relationship in our model, we compute $\gamma_{\mathrm{FB}}$ in the analytically convenient case where $\Delta \tau$ is small.

Proposition 2 (Positive Premia-Slope Relationship). For $\Delta \tau \rightarrow 0$ and for all $\tau$, the regression coefficient in (21) is $\gamma_{F B}=\frac{\kappa_{r}^{*}-\kappa_{r}}{\kappa_{r}^{*}}>0$.

The negative relationship between bond risk premia and the short rate is reflected in the behavior of forward rates. Suppose, for example, that the short rate is high, in which case arbitrageurs

\footnotetext{
${ }^{15}$ The assumption that the demand of preferred-habitat investors is elastic in the yield of the bond corresponding to their maturity habitat is crucial for this result. Indeed, if the demand were inelastic, arbitrageurs would not trade with preferred-habitat investors. Therefore their positions would be independent of the short rate, and so would bond risk premia.
} 
are short bonds and are subject to the risk that rates will decrease. Arbitrageurs are compensated for that risk by the negative expected returns that bonds offer relative to the short rate. Bonds are thus expensive and their yields are low compared to the expectations-hypothesis (EH) benchmark where expected excess returns are zero. Since forward rates are increasing in bond yields, they are also low compared to the EH benchmark, i.e., they are below expected spot rates. Conversely, forward rates exceed expected spot rates when the short rate is low. Forward rates thus under-react to changes in expected spot rates: they increase less than expected spot rates following positive shocks to the current short rate and decrease less following negative shocks. ${ }^{16} \mathrm{~A}$ related explanation for under-reaction, which is useful in later sections, is that in the absence of arbitrageurs forward rates are independent of expected short rates. Arbitrageurs incorporate information about expected short rates into forward rates, but their activity is limited by risk aversion. As a result, information is not fully incorporated, implying under-reaction. Proposition 3 confirms under-reaction by comparing how a shock to the short rate $r_{t}$ impacts the expected short rate at time $t+\tau$ and the instantaneous forward rate $f_{t, \tau}$ for maturity $\tau$. The latter rate is defined as the limit of the forward rate $f_{t, \tau-\Delta \tau, \tau}$ between maturities $\tau-\Delta \tau$ and $\tau$ when $\Delta \tau$ goes to zero:

$$
f_{t, \tau} \equiv \lim _{\Delta \tau \rightarrow 0} f_{t, \tau-\Delta \tau, \tau}=-\frac{\partial \log \left(P_{t, \tau}\right)}{\partial \tau}=A_{r}^{\prime}(\tau) r_{t}+C^{\prime}(\tau)
$$

where the second step follows from (2), and the third from (9).

Proposition 3 (Under-Reaction of Forward Rates). A unit shock to the short rate $r_{t}$

- Raises the expected short rate at time $t+\tau$ by $\frac{\partial E_{t}\left(r_{t+\tau}\right)}{\partial r_{t}}=e^{-\kappa_{r} \tau}$.

- Raises the instantaneous forward rate $f_{t, \tau}$ for maturity $\tau$ by $\frac{\partial f_{t, \tau}}{\partial r_{t}}=e^{-\kappa_{r}^{*} \tau}<e^{-\kappa_{r} \tau}$.

The properties of bond risk premia and forward rates are reflected in the risk-neutral parameters $\left(\bar{r}^{*}, \kappa_{r}^{*}\right)$ and the market price of short-rate risk $\lambda_{r, t}$. Eq. (18) implies that the short rate mean-reverts

\footnotetext{
${ }^{16}$ The under-reaction of forward rates to changes in expected spot rates is relevant in the context of recent events. For example, short-rate cuts triggered by the 2007-2008 financial crisis rendered the US term structure steeply upward sloping. According to a Barclays Capital report by Pradhan (2009), p.2., forward rates did not decrease enough to reflect the low expected future spot rates, i.e., the forward-rate curve remained too flat. This point is made in the context of the two-year rate: while the two-year spot rate is $258 \mathrm{bps}$ lower than the ten-year spot rate, the difference between the same rates two years forward is only $93 \mathrm{bps}$.

... In our opinion, forward steepeners still offer value; for instance, the $2 \mathrm{~s}-10$ s swap curve $2 \mathrm{y}$ forward is trading at $93 \mathrm{bp}$ versus the spot level of $258 \mathrm{bp}$. The flatness of the forward curve largely reflects the market pricing in aggressive Fed hikes, which we believe understates the severe depth of the current recession and its effect on the path of the fed funds rate...

The proposed strategy is to lend at the two-year rate two years forward and borrow at the ten-year rate two years forward. Lending at the two-year rate two years forward is a simple carry roll-down trade: it amounts to shorting two-year bonds and buying four-year bonds. Adding the position in the ten-year rate two years forward makes the trade a butterfly. Such butterfly trades are consistent with our model, as we explain in Footnote 21.
} 
faster under the risk-neutral than under the true measure $\left(\kappa_{r}^{*}>\kappa_{r}\right.$, a property that is used to derive Propositions 2 and 3). To explain the intuition, suppose, for example, that the short rate is high, in which case bond risk premia are negative and bond yields are low compared to the EH benchmark. Since the EH holds under the risk-neutral measure, spot rates are expected to drop faster than under the true measure, meaning that mean-reversion is faster.

Eq. (14) implies that $\lambda_{r, t}$ is an affine and decreasing function of the short rate $r_{t}$. Therefore, $\lambda_{r, t}$ mirrors the behavior of bond risk premia, being negative when $r_{t}$ is large and positive when $r_{t}$ is small. This functional form of $\lambda_{r, t}$ has been proposed by Dai and Singleton (DS 2002) and Duffee (2002) in the context of reduced-form modeling, and as part of the broader class of essentially-affine specifications. ${ }^{17}$ We show that this functional form arises in an equilibrium model with segmented maturity markets integrated by limited arbitrage. Moreover, because our model is equilibrium rather than reduced form, we can give economic interpretations to term-structure factors, and relate properties of the term structure to variables outside the set of bond yields, such as demand and supply, and arbitrageur risk aversion. We examine the effects of demand and supply in later sections, where we introduce demand risk factors. ${ }^{18}$ Corollary 1 shows that an increase in arbitrageur risk aversion strengthens the positive relationship between bond risk premia and the slope of the term structure, as well as the under-reaction of forward rates to changes in expected spot rates. The intuition is that both effects arise because of risk aversion: when arbitrageurs are risk neutral, the EH holds, bond risk premia are zero and forward rates react one-for-one to changes in expected spot rates.

Corollary 1. When arbitrageurs are more risk averse (larger a):

- The regression coefficient $\gamma_{F B}$ of bond excess returns on term-structure slope, derived in Proposition 2, is larger.

- The under-reaction of forward rates to changes in expected spot rates, derived in Proposition 3 , is stronger.

Corollary 1 is a comparative-statics result because arbitrageur risk aversion $a$ is constant in our model. Stepping outside of the model, however, we can interpret the corollary as concerning

\footnotetext{
${ }^{17} \mathrm{DS}$ and Duffee show that specifications in which market prices of risk do not change sign, such as the one arising in the equilibrium model of Cox, Ingersoll and Ross (1985), fail to match important properties of the data. They propose the class of essentially affine specifications, in which market prices of risk are affine functions of the state variables. These specifications generate a better match with the data, while retaining the tractability of affine models.

${ }^{18}$ When the short rate is the only risk factor, demand and supply manifest themselves through the parameter $\bar{\beta}$, which determines the level of the flat term structure in the absence of arbitrageurs. The parameter $\bar{\beta}$ affects the term structure through the long-run mean $\bar{r}^{*}$ of the short rate under the risk-neutral measure (Eq. (19)).
} 
the effects of time-variation in $a$. If, for example, $a$ is decreasing in arbitrageur capital, then timevariation in $a$ could be measured by arbitrageur returns. Empirical proxies for the latter are the returns of hedge funds or the profit-loss positions of proprietary-trading desks. But our model suggests even more direct proxies (in the sense of requiring only term-structure data), derived from arbitrageurs' trading strategies. For example, at times when the term structure is upward sloping, our model predicts that arbitrageurs are engaged in the carry roll-down trade. Therefore, arbitrageur capital decreases when that trade loses money. Conversely, when the term structure is downward sloping, arbitrageurs are engaged in the carry roll-up trade, and their capital decreases when that trade loses money. Using proxies for arbitrageur capital based on this idea, Greenwood and Vayanos (2009a) find empirical support for the predictions of our model.

Our analysis offers a new perspective into the transmission of monetary policy. Suppose that the Central Bank conducts monetary policy through the short rate. The effects of this policy are propagated along the term structure through the carry trades of arbitrageurs, and more broadly of intermediaries engaging in maturity transformation, such as banks. This propagation mechanism is effective when arbitrageur risk aversion is low, e.g., arbitrageurs are well capitalized. When, however, risk aversion is high, propagation is less effective and forward rates are not responsive to changes in expected short rates (Corollary 1). In such circumstances, direct intervention into the markets for long-term bonds might be more effective in influencing long rates. ${ }^{19}$

In acting as conduits of monetary policy, arbitrageurs reap rewards in the form of positive expected returns from their carry trades. Thus, monetary policy transfers wealth to arbitrageurs in expected terms. For example, a policy that keeps the short rate low, and the term structure upward sloping, transfers wealth through positive bond risk premia. The magnitude of this transfer is small when arbitrageurs are not very risk averse because they compete away the premia, but can be large when risk aversion is high.

\section{Two-Factor Model}

This section studies the case where there is one demand factor $(K=1)$. For notational convenience we omit the factor subscript from quantities pertaining to that factor, e.g., use $\beta_{t}$ instead of $\beta_{1, t}$. The term structure is described by a model with two factors: the short rate $r_{t}$ and demand $\beta_{t}$.

\footnotetext{
${ }^{19}$ While we explore the effects of direct intervention in later sections, a full analysis of how to best influence long rates is beyond the scope of our model.
} 


\subsection{Equilibrium}

We conjecture that equilibrium spot rates are affine in $\left(r_{t}, \beta_{t}\right)$, i.e.,

$$
P_{t, \tau}=e^{-\left[A_{r}(\tau) r_{t}+A_{\beta}(\tau) \beta_{t}+C(\tau)\right]}
$$

for three functions $A_{r}(\tau), A_{\beta}(\tau), C(\tau)$ that depend on maturity $\tau$. Applying Ito's Lemma to (23) and using the dynamics (3) of $r_{t}$ and (6) of $\beta_{t}$, we find that the instantaneous return on the bond with maturity $\tau$ is

$$
\frac{d P_{t, \tau}}{P_{t, \tau}}=\mu_{t, \tau} d t-A_{r}(\tau) \sigma_{r} d B_{r, t}-A_{\beta}(\tau) \sigma_{\beta} d B_{\beta, t},
$$

where

$$
\mu_{t, \tau} \equiv A_{r}^{\prime}(\tau) r_{t}+A_{\beta}(\tau) \beta_{t}+C^{\prime}(\tau)-A_{r}(\tau) \kappa_{r}\left(\bar{r}-r_{t}\right)+A_{\beta}(\tau) \kappa_{\beta} \beta_{t}+\frac{1}{2} A_{r}(\tau)^{2} \sigma_{r}^{2}+\frac{1}{2} A_{\beta}(\tau)^{2} \sigma_{\beta}^{2}
$$

is the instantaneous expected return. Substituting (24) into the arbitrageurs' budget constraint (7), we can solve the arbitrageurs' optimization problem (8).

Lemma 2. The arbitrageurs' first-order condition is

$$
\mu_{t, \tau}-r_{t}=A_{r}(\tau) \lambda_{r, t}+A_{\beta}(\tau) \lambda_{\beta, t}
$$

where

$$
\begin{aligned}
& \lambda_{r, t} \equiv a \sigma_{r}^{2} \int_{0}^{T} x_{t, \tau} A_{r}(\tau) d \tau, \\
& \lambda_{\beta, t} \equiv a \sigma_{\beta}^{2} \int_{0}^{T} x_{t, \tau} A_{\beta}(\tau) d \tau .
\end{aligned}
$$

Eq. (26) requires that a bond's expected excess return $\mu_{t, \tau}-r_{t}$ is a linear function of the bond's sensitivities $A_{r}(\tau)$ to the short rate and $A_{\beta}(\tau)$ to the demand factor. The coefficients $\lambda_{r, t}$ and $\lambda_{\beta, t}$ of the linear function (which are the same for all bonds) are the market prices of short-rate and demand risk, respectively. Eq. (26) is the two-factor counterpart of (12), and follows solely from the absence of arbitrage: the expected excess return per unit of each type of risk must be the same for all bonds.

The market prices of risk $\lambda_{r, t}$ and $\lambda_{\beta, t}$ are the expected excess returns that arbitrageurs require as compensation for taking a marginal unit of short-rate and demand risk, respectively. They are 
linear and increasing in the covariance with the arbitrageurs' portfolio. Since arbitrageurs' positions in equilibrium are opposite to those of preferred-habitat investors, and the latter are affine in spot rates, $\lambda_{r, t}$ and $\lambda_{\beta, t}$ are affine in $\left(r_{t}, \beta_{t}\right)$. Substituting $\left(\mu_{t, \tau}, \lambda_{r, t}, \lambda_{\beta, t}\right)$ into (26), we find an affine equation in $\left(r_{t}, \beta_{t}\right)$. Setting linear terms in $\left(r_{t}, \beta_{t}\right)$ to zero yields

$$
\begin{aligned}
& A_{r}^{\prime}(\tau)+\kappa_{r} A_{r}(\tau)-1=A_{r}(\tau) M_{1,1}+A_{\beta}(\tau) M_{1,2}, \\
& A_{\beta}^{\prime}(\tau)+\kappa_{\beta} A_{\beta}(\tau)=A_{r}(\tau) M_{2,1}+A_{\beta}(\tau) M_{2,2},
\end{aligned}
$$

where

$$
M \equiv a\left[\begin{array}{cc}
-\sigma_{r}^{2} \int_{0}^{T} \alpha(\tau) A_{r}(\tau)^{2} d \tau & -\sigma_{\beta}^{2} \int_{0}^{T} \alpha(\tau) A_{r}(\tau) A_{\beta}(\tau) d \tau \\
\sigma_{r}^{2} \int_{0}^{T} \alpha(\tau)\left[\tau \theta(\tau)-A_{\beta}(\tau)\right] A_{r}(\tau) d \tau & \sigma_{\beta}^{2} \int_{0}^{T} \alpha(\tau)\left[\tau \theta(\tau)-A_{\beta}(\tau)\right] A_{\beta}(\tau) d \tau
\end{array}\right]
$$

and setting constant terms to zero yields an equation for $C(\tau)$. Eqs. (29) and (30) constitute a system of two linear differential equations in $A_{r}(\tau), A_{\beta}(\tau)$, in which the coefficients of $A_{r}(\tau), A_{\beta}(\tau)$ depend on integrals involving these functions. This is because of the same fixed-point problem arising in the one-factor model: the risk-neutral measure (implicit in the matrix $M$ ) determines the functions $A_{r}(\tau), A_{\beta}(\tau)$, but it also depends on these functions. Proposition 4 reduces the fixed-point problem to a system of four scalar non-linear equations.

Proposition 4. The functions $A_{r}(\tau), A_{\beta}(\tau)$ are given by

$$
\begin{aligned}
& A_{r}(\tau)=\frac{1-e^{-\nu_{1} \tau}}{\nu_{1}}+\gamma_{r}\left(\frac{1-e^{-\nu_{2} \tau}}{\nu_{2}}-\frac{1-e^{-\nu_{1} \tau}}{\nu_{1}}\right), \\
& A_{\beta}(\tau)=\gamma_{\beta}\left(\frac{1-e^{-\nu_{2} \tau}}{\nu_{2}}-\frac{1-e^{-\nu_{1} \tau}}{\nu_{1}}\right)
\end{aligned}
$$

where the scalars $\left(\nu_{1}, \nu_{2}, \gamma_{r}, \gamma_{\beta}\right)$ solve the system of (A.27)-(A.30). The function $C(\tau)$ is given by (A.31).

The system of (A.27)-(A.30), which determines the functions $A_{r}(\tau), A_{\beta}(\tau)$, does not have a simple closed-form solution as in the one-factor model. Nevertheless, closed-form solutions can be derived in the polar cases where arbitrageur risk aversion is very low $(a \approx 0)$ or very high $(a \approx \infty)$. We focus on these cases because they convey useful intuitions, and we complement our analysis by highlighting additional effects derived for intermediate values of risk aversion.

Proposition 5 determines the asymptotic behavior of the parameters $\left(\nu_{1}, \nu_{2}, \gamma_{r}, \gamma_{\beta}\right)$ for $a \approx 0$ and $a \approx \infty$. The proposition reports mainly orders of magnitude (in terms of $a$ ). Full closed-form 
solutions are in the Appendix, and solutions for some of the parameters are reported later in this section when needed to illustrate our results.

Proposition 5. The asymptotic behavior of $\left(\nu_{1}, \nu_{2}, \gamma_{r}, \gamma_{\beta}\right)$ is as follows:

- When $a \approx 0:\left(\nu_{1}, \nu_{2}, \gamma_{r}, \gamma_{\beta}\right) \approx\left(\kappa_{r}, \kappa_{\beta}, a^{3} \underline{c}_{r}, a \underline{c}_{\beta}\right)$, where $\underline{c}_{r}$ and $\underline{c}_{\beta}$ are constants given by (A.39) and (A.38), respectively.

- When $a \approx \infty:\left(\nu_{1}, \nu_{2}, \gamma_{r}, \gamma_{\beta}\right) \approx\left(a^{\frac{1}{3}} \bar{n}_{1}, \bar{\nu}_{2}, a^{-\frac{1}{3}} \bar{c}_{r}, \bar{\gamma}_{\beta}\right)$, where $\bar{n}_{1}, \bar{\nu}_{2}, \bar{c}_{r}$ and $\bar{\gamma}_{\beta}$ are constants given by (A.49), (A.48), (A.50) and (A.51), respectively. Furthermore, $\bar{n}_{1}>0$ and $\bar{c}_{r}<0$.

\subsection{Term Structure Movements}

In the two-factor model, the term structure moves because of shocks to the short rate and to demand. Examining the impact of these shocks reveals key economic mechanisms of our model and yields new results. We examine how the shocks affect the term structure of instantaneous forward rates rather than of spot rates. Recall that the instantaneous forward rate for maturity $\tau$ is the limit of the forward rate $f_{t, \tau-\Delta \tau, \tau}$ between maturities $\tau-\Delta \tau$ and $\tau$ when $\Delta \tau$ goes to zero:

$$
f_{t, \tau} \equiv \lim _{\Delta \tau \rightarrow 0} f_{t, \tau-\Delta \tau, \tau}=-\frac{\partial \log \left(P_{t, \tau}\right)}{\partial \tau}=A_{r}^{\prime}(\tau) r_{t}+A_{\beta}^{\prime}(\tau) \beta_{t}+C^{\prime}(\tau),
$$

where the second step follows from (2), and the third from (23). Since the spot rate for maturity $\tau$ is the average of instantaneous forward rates up to that maturity (from (1) and (34)), the term structure of instantaneous forward rates conveys the same information as that of spot rates. We use instantaneous forward rates because they are directly comparable to expected short rates. ${ }^{20}$ Eq. (34) implies that the impact of short-rate and demand shocks on instantaneous forward rates is characterized by the functions $A_{r}^{\prime}(\tau)$ and $A_{\beta}^{\prime}(\tau)$, respectively.

When arbitrageurs are close to risk-neutral $(a \approx 0)$, Propositions 4 and 5 imply that a unit shock to the short rate $r_{t}$ raises the instantaneous forward rate for maturity $\tau$ by

$$
A_{r}^{\prime}(\tau) \approx e^{-\kappa_{r} \tau}
$$

The effect is approximately the same as on the corresponding expected short rate. This is because the expectations hypothesis $(\mathrm{EH})$ holds when arbitrageurs are risk neutral.

\footnotetext{
${ }^{20}$ Suppose, for example, that the 29- and 30 -year spot rates are $4 \%$ and $3.88 \%$, respectively. The forward rate between maturities 29 and 30 is $0.46 \%$, and can appear low to an arbitrageur in comparison to the expected one-year spot rate in 29 years. Such a comparison, however, is not as transparent when looking at the 29- and 30-year spot rates.
} 
Under the EH, the term structure is determined solely by current and expected future short rates. Therefore, demand shocks have no effect when arbitrageurs are risk neutral. Examining the effect of demand shocks close to risk neutrality, however, yields useful insights. Propositions 4 and 5 imply that when $a \approx 0$, a unit shock to the demand factor $\beta_{t}$ raises the instantaneous forward rate for maturity $\tau$ by

$$
A_{\beta}^{\prime}(\tau) \approx \frac{a \sigma_{r}^{2} \int_{0}^{T} \alpha(\tau) \tau \theta(\tau) \frac{1-e^{-\kappa_{r} \tau}}{\kappa_{r}} d \tau}{\kappa_{r}-\kappa_{\beta}}\left(e^{-\kappa_{\beta} \tau}-e^{-\kappa_{r} \tau}\right)
$$

To illustrate (36), suppose that $\theta(\tau)$ is positive for a subset $\mathcal{T}$ of maturities, and zero for all others, so an increase in $\beta_{t}$ represents a decrease in demand by investors whose habitats are in $\mathcal{T}$. Eq. (36) implies that $A_{\beta}^{\prime}(\tau)>0$ for all $\tau$, i.e., the decrease in demand raises instantaneous forward rates for all maturities, including those not in $\mathcal{T}$. This is because arbitrageurs accommodate the decrease in demand by buying bonds. Therefore, they become exposed to the risk that bond prices decrease, and require higher returns to hold bonds. This pushes bond prices down and forward rates up.

We next examine how the effect of the demand shock depends on the function $\theta(\tau)$ that characterizes the shock's location $\mathcal{T}$ in the maturity space. The function $\theta(\tau)$ enters in $(36)$ through the integral $\int_{0}^{T} \alpha(\tau) \tau \theta(\tau) \frac{1-e^{-\kappa_{r} \tau}}{\kappa_{r}} d \tau$. The term $\alpha(\tau) \tau \theta(\tau)$ represents the dollar value of the bond with maturity $\tau$ bought by arbitrageurs in response to the shock, and is multiplied by $\frac{1-e^{-\kappa_{r} \tau}}{\kappa_{r}}$, the bond's sensitivity to the short rate. This sensitivity is the counterpart of duration in our model, and the integral $\int_{0}^{T} \alpha(\tau) \tau \theta(\tau) \frac{1-e^{-\kappa_{r} \tau}}{\kappa_{r}} d \tau$ can be interpreted as the duration of the portfolio bought by arbitrageurs. When duration is high, the portfolio is risky, and the demand shock triggers a sharp increase in required returns and forward rates. Note that duration is measured only in reference to changes in the short rate. This is because when arbitrageurs are close to risk-neutral, demand effects are small, and the short rate is the dominant risk factor.

While $\theta(\tau)$ determines the magnitude of the demand effect, it is unrelated to the set of maturities for which the effect is largest. This is because the demand shock affects the term structure by altering the market price of short-rate risk, which is common to all bonds because of the absence of arbitrage. Bonds most heavily affected by the shock are those most sensitive to changes in the market price of short-rate risk, and do not necessarily lie within the shock's location $\mathcal{T}$ in the maturity space. Thus, our model refines and qualifies the logic of limited arbitrage. Consistent with that logic, we find that demand shocks affect prices because arbitrageurs' risk tolerance is limited. Yet, even with limited risk tolerance, arbitrageurs are able to eliminate riskfree arbitrage opportunities. 
As a consequence, arbitrage - even limited - imposes tight restrictions on how demand shocks affect the cross-section of bonds, which is driven by one dominant risk factor. These restrictions become looser when arbitrageurs are concerned with multiple risk factors, but they remain effective as we show later in this section.

The function $\frac{e^{-\kappa_{\beta} \tau}-e^{-\kappa_{r} \tau}}{\kappa_{r}-\kappa_{\beta}}$, which characterizes the relative importance of the demand effect across maturities, is hump-shaped, and equal to zero for $\tau=0$ and $\tau=\infty$. Therefore, the effect of demand shocks is largest for intermediate or long maturities, while that of short-rate shocks (characterized by the function $e^{-\kappa_{r} \tau}$ ) is largest for short maturities. This is because the two types of shocks work through different channels: short-rate shocks affect expected short rates, which are the main determinant of instantaneous forward rates for short maturities, while demand shocks affect risk premia, which become important for longer maturities.

We next examine the effects of short-rate and demand shocks when arbitrageur risk aversion is very high. Propositions 4 and 5 imply that when $a \approx \infty$, a unit shock to the short rate $r_{t}$ raises the instantaneous forward rate for maturity $\tau$ by

$$
A_{r}^{\prime}(\tau) \approx e^{-a^{\frac{1}{3}} \bar{n}_{1} \tau}+a^{-\frac{1}{3}} \bar{c}_{r} e^{-\bar{\nu}_{2} \tau}
$$

The first term in (37) represents an under-reaction of forward rates to changes in expected short rates, also present in the one-factor model. Recall that under-reaction arises because information about expected short rates is incorporated into forward rates through arbitrageurs' carry trades, which are limited because of risk aversion. When arbitrageurs are very risk averse, under-reaction is extreme: information about expected short rates is incorporated into forward rates only for short maturities because carry trades for long maturities involve long-term bonds and therefore high risk. The extreme under-reaction of instantaneous forward rates for long maturities is reflected into the first term in (37), which is positive but dies off to zero quickly (rate $a^{\frac{1}{3}} \bar{n}_{1}$ ) as maturity increases.

The second term in (37) represents a new effect arising in the two-factor model. Since $\bar{c}_{r}<0$, this term is negative. Moreover, because it dies off to zero more slowly (rate $\bar{\nu}_{2}$ ) than the first term, it becomes dominant for long maturities. As a consequence, instantaneous forward rates for long maturities move in the opposite direction to the short-rate shock (and to expected short rates). This effect arises because of arbitrageurs' hedging activity. Recall that in response to a positive short-rate shock, arbitrageurs invest at the short rate and short bonds. When, in addition, arbitrageurs are very risk averse, they limit their shorting activity to short-term bonds. Moreover, because long-term bonds are highly sensitive to demand risk, arbitrageurs not only refrain from 
shorting them, but instead buy them to hedge the demand risk of their short positions in short-term bonds. The effect of this "butterfly" trade is to push prices of long-term bonds up and long rates down. ${ }^{21}$

Corollary 2. When $a \approx \infty$, a shock to the short rate $r_{t}$ moves instantaneous forward rates in the same direction for short maturities but in the opposite direction for long maturities. The effect on spot rates exhibits the same pattern.

Propositions 4 and 5 imply that when $a \approx \infty$, a unit shock to the demand factor $\beta_{t}$ raises the instantaneous forward rate for maturity $\tau$ by

$$
A_{\beta}^{\prime}(\tau) \approx \frac{\int_{0}^{T} \alpha(\tau) \tau \theta(\tau) \frac{1-e^{-\bar{\nu}_{2} \tau}}{\bar{\nu}_{2}} d \tau}{\int_{0}^{T} \alpha(\tau)\left(\frac{1-e^{-\bar{\nu}_{2} \tau}}{\bar{\nu}_{2}}\right)^{2} d \tau}\left(e^{-\bar{\nu}_{2} \tau}-e^{-a^{\frac{1}{3}} \bar{n}_{1} \tau}\right)
$$

The demand effect in (38) has similarities with its counterpart for $a \approx 0$ in (36), but there are also important differences. Suppose that $\theta(\tau)$ is positive for a subset $\mathcal{T}$ of maturities, and zero for all others, so an increase in $\beta_{t}$ represents a decrease in demand by investors whose habitats are in $\mathcal{T}$. As in the case $a \approx 0$, the decrease in demand raises instantaneous forward rates for all maturities. Moreover, the effect is largest for intermediate maturities (if $\bar{\nu}_{2}<0$ ) or long maturities (if $\bar{\nu}_{2}>0$ ), while the effect of short-rate shocks is largest for short maturities.

A key difference with the case $a \approx 0$ is that $\theta(\tau)$ influences not only the magnitude of the demand effect, but also its relative importance across maturities. When $a \approx 0$, absence of arbitrage and the approximate one-factor structure impose tight restrictions on how the demand shock affects the cross-section of bonds. To explain the intuition when $a \approx \infty$, we start with a hypothetical extreme case where there is a large number of risk factors. If the demand effect is largest for a subset $\mathcal{T}^{\prime}$ of maturities disjoint from the shock's location $\mathcal{T}$, this means that arbitrageurs buy bonds with maturities in $\mathcal{T}$ and short bonds with maturities in $\mathcal{T}^{\prime}$. When arbitrageurs are very risk averse, this portfolio must have zero exposure to all factors, which is infeasible with a large number of factors. Thus, arbitrageurs are unwilling to integrate maturity markets, and the demand effect remains localized at $\mathcal{T}$.

\footnotetext{
${ }^{21}$ Butterfly trades consist of positions in three maturities: two positions of the same sign at the extremes and a position of the opposite sign at the middle. Such trades are common in term-structure arbitrage. Returning to the example in Footnote 16, the trade proposed in Pradhan (2009) is to lend at the two-year rate two years forward and borrow at the ten-year rate two years forward. The first leg of this trade amounts to shorting two-year bonds and buying four-year bonds, while the second leg amounts to buying two-year bonds and shorting twelve-year bonds. The second leg serves to hedge term-structure movements at intermediate maturities, and is for a smaller notional amount since the twelve-year bond is more sensitive to such movements than the four-year bond. Therefore, the overall trade amounts to shorting two-year bonds, buying four-year bonds, and shorting twelve-year bonds, i.e., a butterfly. This puts upward pressure on the twelve-year spot rate, despite the trade being triggered by a rate drop at the short end of the term structure.
} 
Formally, suppose that arbitrageurs start with a zero position in every bond. A unit shock to the demand factor $\beta_{t}$ induces them to buy dollar value $\alpha(\tau)\left[\tau \theta(\tau)-A_{\beta}(\tau)\right]$ of the bond with maturity $\tau .{ }^{22}$ This generates portfolio exposures

$$
\begin{aligned}
& \int_{0}^{T} \alpha(\tau)\left[\tau \theta(\tau)-A_{\beta}(\tau)\right] A_{r}(\tau) d \tau, \\
& \int_{0}^{T} \alpha(\tau)\left[\tau \theta(\tau)-A_{\beta}(\tau)\right] A_{\beta}(\tau) d \tau,
\end{aligned}
$$

to the factors $r_{t}$ and $\beta_{t}$, respectively. When arbitrageurs are very risk averse, they seek to set these exposures to zero. If a large number of factors are added to $\left(r_{t}, \beta_{t}\right)$, the zero-exposure requirement becomes very strict: integrals of the function $\tau \theta(\tau)-A_{\beta}(\tau)$ times a large number of different functions must be zero. A natural conjecture, to which we return in Section 5, is that the function $\tau \theta(\tau)-A_{\beta}(\tau)$ must be zero for all $\tau$. This means that arbitrageurs hold zero positions in all bonds, and the effect $A_{\beta}(\tau) / \tau$ of a demand shock on the spot rate is equal to the function $\theta(\tau)$ that characterizes the shock's location in the maturity space. ${ }^{23}$

With two factors only, the zero-exposure requirement amounts to two scalar equations, and does not imply that $\tau \theta(\tau)-A_{\beta}(\tau)$ is zero for all $\tau$. In fact, $A_{\beta}(\tau)$ cannot coincide with a general function $\tau \theta(\tau)$ because absence of arbitrage and the two-factor structure restrict it to be an affine function of two exponentials. The function $\theta(\tau)$, however, influences the variation of $A_{\beta}(\tau)$ across maturities because it affects $\bar{\nu}_{2}$. Proposition 6 shows that a shift in the demand shock's location towards longer maturities raises the maturity at which the shock's effect $A_{\beta}^{\prime}(\tau)$ is largest, from intermediate to long.

Proposition 6. Suppose that $a \approx \infty$, and consider a family of positive functions $\{\theta(\tau, x)\}_{x \in(\underline{x}, \bar{x})}$ such that

- The function $\theta_{x}(\tau, x) / \theta(\tau, x)$ is increasing in $\tau$ (i.e., higher values of $x$ shift weight towards longer maturities).

- For all $\epsilon>0, \lim _{x \rightarrow \underline{x}} \frac{\int_{\epsilon}^{T} \alpha(\tau) \tau \theta(\tau, x) d \tau}{\int_{0}^{T} \alpha(\tau) \tau \theta(\tau, x) d t}=0$ and $\lim _{x \rightarrow \bar{x}} \frac{\int_{0}^{T-\epsilon} \alpha(\tau) \tau \theta(\tau, x) d \tau}{\int_{0}^{T} \alpha(\tau) \tau \theta(\tau, x) d t}=0$ (i.e., $\theta(\tau, x)$ gives all weight to $\tau=0$ when $x \rightarrow \underline{x}$ and to $\tau=T$ when $x \rightarrow \bar{x})$.

\footnotetext{
${ }^{22}$ Eq. $\partial x_{t, \tau} / \partial \beta_{t}=\alpha(\tau)\left[\tau \theta(\tau)-A_{\beta}(\tau)\right]$ follows from $x_{t, \tau}=-y_{t, \tau},(1),(4),(5)$ and (23).

${ }^{23}$ The assumption that the demand of preferred-habitat investors is elastic in the yield of the bond corresponding to their maturity habitat is crucial for the argument. Indeed, if investors were buying or selling bonds inelastically, market-clearing would require arbitrageurs to hold non-zero positions.
} 
Then, there exists $x^{*} \in(\underline{x}, \bar{x})$ such that $A_{\beta}^{\prime}(\tau)$ is increasing and then decreasing in $[0, T]$ if $x<x^{*}$, and is increasing in $[0, T]$ if $x>x^{*}$.

Gathering results from $a \approx 0$ and $a \approx \infty$ yields the following corollary.

Corollary 3 (Preferred Habitat). A decrease in demand by preferred-habitat investors raises the term structure of instantaneous forward rates and of spot rates.

- When $a \approx 0$, the demand shock's location in the maturity space influences the magnitude of the demand effect but not its relative importance across maturities.

- When $a \approx \infty$, the demand shock's location in the maturity space influences both the magnitude of the demand effect and its relative importance across maturities. In particular, a shift in the shock's location towards longer maturities raises the maturity at which the effect is largest, from intermediate to long.

When $a$ is not close to 0 or $\infty$, the analysis of term structure movements becomes more complicated because the system of (A.27)-(A.30) does not have a closed-form solution. In a parameter region where $\kappa_{r}>\kappa_{\beta}$, i.e., the short-rate mean-reverts faster than the demand factor, the solution has similar qualitative features for small and large values of $a$. In particular, $\nu_{1}>\nu_{2}$ and $\gamma_{r}<0$, implying that a short-rate shock moves instantaneous forward rates in the opposite direction for long maturities. ${ }^{24}$ In a different parameter region where $\kappa_{r}<\kappa_{\beta}$, the same result holds only for large values of $a$, while for small values all forward rates move in the same direction as the shock. Moreover, for intermediate values of $a$, the solution to (A.27)-(A.30) can be complex, meaning that the functions $A_{r}(\tau), A_{\beta}(\tau)$ exhibit oscillation. This is because of the interplay between arbitrageurs' hedging activity and price volatility. Recall from Corollary 2 that prices of short-term bonds comove negatively with the short rate, but comovement can be positive for long-term bonds because arbitrageurs use them to hedge demand risk. Because of the positive comovement, expected returns of bonds at even longer maturities do not increase as much when the market price of short-rate risk increases, implying that these bonds are not as sensitive to demand risk. As a consequence, arbitrageurs do not use these bonds to hedge demand risk, and their prices can comove negatively with the short rate (which affects expected short rates far in the future when $\kappa_{r}$ is small), leading to an oscillating pattern. We leave a detailed study of these effects for future work.

We finally draw the connection between our analysis of term structure movements and analyses based on principal components (PCs). Empirical models often describe movements in terms of

\footnotetext{
${ }^{24}$ The cutoff maturity below which forward rates move in the same direction as the short-rate shock goes to $\infty$ when $a$ goes to zero.
} 
three PCs: level, slope and curvature. The level PC is flat across maturities and explains about $90 \%$ of variation in bond returns. Adding slope and curvature explains almost $100 \%$ of variation (Litterman and Scheinkman (1991)). In our two-factor model, the term structure moves because of shocks to the short rate and to demand. These economic factors are independent, and their relative impact varies across maturities: short-rate shocks impact mainly short maturities, while demand shocks impact mainly long maturities. Yet, for most parametrizations of the model, the first PC explains more than $90 \%$ of variation (and adding the second explains $100 \%$ because there are only two factors). The first PC is a weighted average of short rate and demand, and can be flat across maturities depending on the parametrization. ${ }^{25}$ Thus, while one PC explains most of the variation in bond returns, this does not mean that returns are generated by one main economic factor. Moreover, giving economic interpretations to PCs can be misleading, since they can be linear combinations of underlying economic factors.

\subsection{Bond Risk Premia}

Bond risk premia depend on the short rate $r_{t}$ and the demand factor $\beta_{t}$. The dependence on $r_{t}$ is because of the same mechanism as in the one-factor model: premia are negative when $r_{t}$ is high so that arbitrageurs are induced to engage in the carry roll-up trade, while premia are positive when $r_{t}$ is low. The dependence on $\beta_{t}$ is what induces arbitrageurs to accommodate changes in demand. Suppose, for example, that $\theta(\tau)$ is positive, so an increase in $\beta_{t}$ represents a decrease in demand. Premia are then positive when $\beta_{t}$ is high so that arbitrageurs are induced to buy bonds and accommodate the decrease in demand, while premia are negative when $\beta_{t}$ is low. Proposition 7 confirms these comparative statics using the closed-form solutions for $a \approx 0$ and $a \approx \infty$. The proposition shows additionally that the market prices of short-rate and demand risk share the same comparative statics as the premia.

Proposition 7. Suppose that $\theta(\tau)>0$ for all $\tau$, and $a \approx 0$ or $a \approx \infty$.

- The instantaneous expected excess return $\mu_{t, \tau}-r_{t}$ on the bond with maturity $\tau$ is decreasing in $r_{t}$, increasing in $\beta_{t}$, and affine in both variables.

\footnotetext{
${ }^{25}$ Consider the following parametrization. Bond maturities go up to $T=30$ years. Mean-reversion and diffusion parameters are $\kappa_{r}=\kappa_{\beta}=0.15$ and $\sigma_{r}=\sigma_{\beta}=0.02$, respectively, meaning that short-rate and demand shocks have a half-life of $\log (2) / 0.15=4.62$ years and annual standard deviation $2 \%$. (The values of $\left(\kappa_{r}, \sigma_{r}\right)$ are consistent with Chan, Karolyi, Longstaff and Sanders (1992).) The function $\theta(\tau)$ is equal to 1 for all $\tau$, meaning that demand shocks affect all maturities equally in the absence of arbitrageurs. Finally, $\alpha(\tau)=\alpha e^{-0.1 \tau}$ and $a \alpha=1.5$. A PC decomposition of instantaneous returns on the sixty bonds with maturities $\tau=0.5,1, . ., 29.5,30$ years yields a first PC that explains $94.61 \%$ of variation and is flat across maturities. The flat PC obscures the different role played by short-rate and demand shocks: short-rate shocks explain $99.92 \%$ of variation for the one-year bond, $92.20 \%$ for the five-year bond, $65.22 \%$ for the ten-year bond, $18.50 \%$ for the twenty-year bond, and $4.79 \%$ for the thirty-year bond. The first PC is the weighted average $0.44 r_{t}+\beta_{t}$ of short rate and demand.
} 
- The market prices $\lambda_{r, t}$ of short-rate risk and $\lambda_{\beta, t}$ of demand risk are decreasing in $r_{t}$, increasing in $\beta_{t}$, and affine in both variables.

Proposition 7 suggests that the positive relationship between bond risk premia and slope of the term structure, derived in the one-factor model, should carry over to two factors. Indeed, the term structure is upward sloping when the short rate is low, or when bond prices are low because demand is low. In both cases, bonds earn positive risk premia. Proposition 8 confirms the positive premia-slope relationship in the context of the Fama-Bliss (FB) regression (21) with small $\Delta \tau$. The proposition shows additionally that the coefficient $\gamma_{\mathrm{FB}}$ of this regression is increasing in the variance of the demand factor.

Proposition 8. Suppose that $a \approx 0$ or $a \approx \infty$. For $\Delta \tau \rightarrow 0$ and for all $\tau$, the regression coefficient $\gamma_{F B}$ in (21) is positive and increasing in $\sigma_{\beta}^{2} / \sigma_{r}^{2}$.

The intuition why the premia-slope relationship is stronger in the presence of the demand factor is that in contrast to the short rate, demand affects slope only through its effect on premia. For example, a decrease in demand generates an upward sloping term structure because it triggers an increase in premia and therefore a decrease in bond prices. An upward sloping term structure is also generated by a decrease in the short rate, but this does not require a change in premia: it happens simply because the current short rate moves below expected future short rates. That the decrease in the short rate triggers an increase in premia only accentuates the increase in slope. Therefore, an increase in slope predicts a larger increase in premia when caused by demand than when caused by the short rate. Greenwood and Vayanos (2009a) build on this idea to show that an increase in bond supply raises premia even controlling for slope, and find empirical support for this prediction.

The regression coefficient $\gamma_{\mathrm{FB}}$ estimated by $\mathrm{FB}$ is approximately equal to one. In our one-factor model, $\gamma_{\mathrm{FB}}$ is equal to $\frac{\kappa_{r}^{*}-\kappa_{r}}{\kappa_{r}^{*}}$, and therefore varies from zero when arbitrageurs are risk neutral, to one when they are infinitely risk averse. In the presence of the demand factor, $\gamma_{\mathrm{FB}}$ is larger, and varies from zero to a value larger than one.

While our model predicts a positive premia-slope relationship, it also yields finer implications on how premia are related to the shape of the term structure. Indeed, the underlying drivers of premia are the short rate $r_{t}$ and the demand factor $\beta_{t}$. Slope, defined in the spirit of FB as the difference between forward rates and the short rate, is an imperfect measure of $\left(r_{t}, \beta_{t}\right)$, and therefore might not be the optimal predictor of premia. Consider, for example, the case where a decrease in 
demand has its maximum impact at intermediate maturities and generates a hump-shaped term structure. Measuring demand based on the hump is then more informative than only looking at the slope. This suggests a link between our analysis and Cochrane and Piazzesi (CP 2005), who find that bond risk premia are best predicted by a tent-shaped combination of forward rates. Their tent-shaped factor is positive when the term structure exhibits a hump, and in that case positive premia are predicted. Such a hump could arise in our model in response to a decrease in demand, and would predict positive premia consistent with CP. A fuller analysis of this issue would require a calibration of our model, possibly in its multi-factor version, and is left for future work.

The behavior of bond risk premia is related to the predictability of long-rate changes. Campbell and Shiller (CS 1991) find that the slope of the term structure predicts changes in long rates, but to a weaker and typically opposite extent than implied by the Expectations Hypothesis (EH). Their regression is

$$
R_{t+\Delta \tau, \tau-\Delta \tau}-R_{t, \tau}=\alpha_{\mathrm{CS}}+\gamma_{\mathrm{CS}} \frac{\Delta \tau}{\tau-\Delta \tau}\left(R_{t, \tau}-R_{t, \Delta \tau}\right)+\epsilon_{t+\Delta \tau}
$$

The dependent variable is the change, between times $t$ and $t+\Delta \tau$, in the yield of a zero-coupon bond that has maturity $\tau$ at time $t$. The independent variable is an appropriately normalized difference between the spot rates for maturities $\tau$ and $\Delta \tau$. According to the $\mathrm{EH}$, the coefficient $\gamma_{\mathrm{CS}}$ should be one. CS find, however, that $\gamma_{\mathrm{CS}}$ is smaller than one, typically negative, and decreasing in $\tau$. This finding is related to the behavior of bond risk premia documented in FB. Indeed, suppose that the term structure is upward sloping. Because bonds earn positive expected excess returns, their yields increase by less than in the EH benchmark, implying a regression coefficient $\gamma_{\mathrm{CS}}$ smaller than one. That $\gamma_{\mathrm{CS}}$ is negative especially for long maturities can be viewed as evidence that premia of longterm bonds are strongly time-varying, e.g., an upward sloping term structure predicts a decrease in long rates because long-term bonds earn very high positive premia. Proposition 9 computes $\gamma_{\mathrm{CS}}$ in our model, in the analytically convenient case where $\Delta \tau$ is small, and shows that it is smaller than one. The proposition shows additionally that when $a \approx \infty, \gamma_{\mathrm{CS}}$ can be negative and decreasing in $\tau$. This decreasing pattern does not arise when $a \approx 0$ (and for any value of $a$ in the one-factor model), and is because the demand factor generates strongly time-varying premia for long-term bonds.

Proposition 9. Suppose that $a \approx 0$ or $a \approx \infty$. For $\Delta \tau \rightarrow 0$ and for all $\tau$, the regression coefficient $\gamma_{C S}$ in (41) is smaller than one. Moreover,

- when $a \approx 0, \gamma_{C S}$ is close to one and increasing in $\tau$.

- when $a \approx \infty, \gamma_{C S}$ can be negative and decreasing in $\tau$. 
The analysis of the two-factor model can easily be extended to the case where the factors are correlated. The effect of correlation is to modify the formulas for the market prices of risk. Denoting the instantaneous correlation between $d B_{r, t}$ and $d B_{\beta, t}$ by $\rho$, and generalizing Lemma 2 , we find

$$
\begin{aligned}
& \lambda_{r, t} \equiv a \sigma_{r}^{2} \int_{0}^{T} x_{t, \tau}\left[A_{r}(\tau)+\rho A_{\beta}(\tau)\right] d \tau \\
& \lambda_{\beta, t} \equiv a \sigma_{\beta}^{2} \int_{0}^{T} x_{t, \tau}\left[\rho A_{r}(\tau)+A_{\beta}(\tau)\right] d \tau .
\end{aligned}
$$

For example, the arbitrageurs' willingness $\lambda_{r, t}$ to take a marginal unit of short-rate risk depends not only on the sensitivity of their portfolio to the short rate but also to the correlated demand factor. Correlation renders the formulas more complicated, but the results in the cases $a \approx 0$ and $a \approx \infty$ remain the same. Indeed, when $a \approx 0$, the short rate is the dominant risk factor, so the correlation between the factors does not matter. When instead $a \approx \infty$, arbitrageurs set the sensitivity of their portfolio to each factor to zero, regardless of the correlation between the factors.

\section{$5 \quad$ Multiple Demand Factors}

In this section we sketch the extension of our analysis to multiple demand factors. We allow for $K>1$ demand factors $\left\{\beta_{k, t}\right\}_{k=1, \ldots, K}$, which together with the short rate $r_{t}$ yield a model with $K+1$ factors. We conjecture that equilibrium spot rates are affine in these factors, i.e.,

$$
P_{t, \tau}=e^{-\left[A_{r}(\tau) r_{t}+\sum_{k=1}^{K} A_{\beta, k}(\tau) \beta_{k, t}+C(\tau)\right]}
$$

for $K+2$ functions $A_{r}(\tau),\left\{A_{\beta, k}(\tau)\right\}_{k=1, . . K}, C(\tau)$ that depend on maturity $\tau$. Generalizing Lemma 2, we find:

Lemma 3. The arbitrageurs' first-order condition is

$$
\mu_{t, \tau}-r_{t}=A_{r}(\tau) \lambda_{r, t}+\sum_{k=1}^{K} A_{\beta, k}(\tau) \lambda_{\beta, k, t}
$$

where $\mu_{t, \tau}$ is given by (A.68),

$$
\begin{aligned}
\lambda_{r, t} & \equiv a \sigma_{r}^{2} \int_{0}^{T} x_{t, \tau} A_{r}(\tau) d \tau, \\
\lambda_{\beta, k, t} & \equiv a \sigma_{\beta}^{2} \int_{0}^{T} x_{t, \tau} A_{\beta, k}(\tau) d \tau .
\end{aligned}
$$


Eq. (43) is the multi-factor counterpart of (12) and (26), and follows solely from the absence of arbitrage. Eqs. (44) and (45) determine the market prices of risk in terms of the arbitrageurs' portfolio. Proceeding as in previous sections, we find that the functions $A_{r}(\tau),\left\{A_{\beta, k}(\tau)\right\}_{k=1, . . K}$ solve a system of $K+1$ linear differential equations, in which the coefficients of $A_{r}(\tau),\left\{A_{\beta, k}(\tau)\right\}_{k=1, . . K}$ depend on integrals involving these functions. Proposition 10 reduces the problem to a system of $(K+1)^{2}$ scalar non-linear equations.

Proposition 10. The functions $A_{r}(\tau),\left\{A_{\beta, k}(\tau)\right\}_{k=1, . . K}$ are given by

$$
\begin{aligned}
A_{r}(\tau) & =\frac{1-e^{-\nu_{1} \tau}}{\nu_{1}}+\sum_{l=1}^{K} \gamma_{r, l}\left(\frac{1-e^{-\nu_{l+1} \tau}}{\nu_{l+1}}-\frac{1-e^{-\nu_{1} \tau}}{\nu_{1}}\right), \\
A_{\beta, k}(\tau) & =\sum_{l=1}^{K} \gamma_{\beta, k, l}\left(\frac{1-e^{-\nu_{l+1} \tau}}{\nu_{l+1}}-\frac{1-e^{-\nu_{1} \tau}}{\nu_{1}}\right)
\end{aligned}
$$

where the scalars $\left\{\nu_{l}\right\}_{l=1, . ., K+1},\left\{\gamma_{r, l}\right\}_{l=1, . ., K},\left\{\gamma_{\beta, k, l}\right\}_{k, l=1, . ., K}$ solve the system of (A.77)-(A.80). The function $C(\tau)$ is given by (A.81).

The system of (A.77)-(A.80), which determines the functions $A_{r}(\tau),\left\{A_{\beta, k}(\tau)\right\}_{k=1, . . K}$, simplifies in the polar cases where arbitrageur risk aversion is very low $(a \approx 0)$ or very high $(a \approx \infty)$. The results when $a \approx 0$ are closely related to their counterparts in the two-factor model. In particular, a unit shock to the demand factor $\beta_{k, t}$ raises the instantaneous forward rate for maturity $\tau$ by

$$
A_{\beta, k}^{\prime}(\tau) \approx \frac{a \sigma_{r}^{2} \int_{0}^{T} \alpha(\tau) \tau \theta_{k}(\tau) \frac{1-e^{-\kappa_{r} \tau}}{\kappa_{r}} d \tau}{\kappa_{r}-\kappa_{\beta, k}}\left(e^{-\kappa_{\beta, k} \tau}-e^{-\kappa_{r} \tau}\right)
$$

which coincides with (36) up to the factor subscript $k$. As in the case of (36), the location of the demand shock in the maturity space determines the magnitude of the demand effect but not the effect's relative importance across maturities. Suppose, for example, that factors $\left(k_{s}, k_{\ell}\right)$ concern demand shocks at short and long maturities, respectively. Because shocks to $k_{\ell}$ impact arbitrageurs' positions in long-term bonds, they have a larger effect, relative to shocks to $k_{s}$, on the duration of arbitrageurs' portfolio. Therefore, they have a larger effect on the market price of short-rate risk $\lambda_{r, t}$ and on the term structure. But if shocks to $k_{\ell}$ mean-revert faster than shocks to $k_{s}$ $\left(\kappa_{\beta, k_{\ell}}>\kappa_{\beta, k_{s}}\right)$, their effect on $\lambda_{r, t}$ is more short-lived, and therefore, their effect on long relative to short maturities is smaller compared to shocks to $k_{s}$. In other words, the relative effect of a demand shock across maturities depends not on the shock's location in the maturity space, but only on the shock's mean-reversion rate. 
The results when $a \approx \infty$ differ from their counterparts in the two-factor model. A natural conjecture, sketched in Section 4.2, is that with a large number of demand factors, demand effects are local to the maturities where shocks originate. Investigating this conjecture requires analyzing a large system of non-linear equations, and is left for future work. ${ }^{26}$ We instead present a numerical example with two demand factors, i.e., a three-factor model, to illustrate the effects of adding factors. For simplicity, we assume that each demand factor concerns shocks at a specific maturity, i.e., the functions $\left\{\theta_{k}(\tau)\right\}_{k=1,2}$ are Dirac. We take the maturities corresponding to factors 1 and 2 to be five and twenty years, respectively, and set $T=30$ years and $\alpha(\tau)=\alpha e^{-0.1 \tau} .{ }^{27}$ Keeping with the notation of the previous paragraph, we denote the "short-maturity" factor 1 by $k_{s}$, and the "long-maturity" factor 2 by $k_{\ell}$. Figure 1 shows the effect of a decrease in demand associated to each factor on the term structure of instantaneous forward rates. The light (red) lines correspond to factor $k_{s}$, and the dark (black) lines to factor $k_{\ell}$. For each factor, the solid line represents the effect of a shock in the three-factor model, and the dashed line represents the same effect in a two-factor model where only that factor is present.

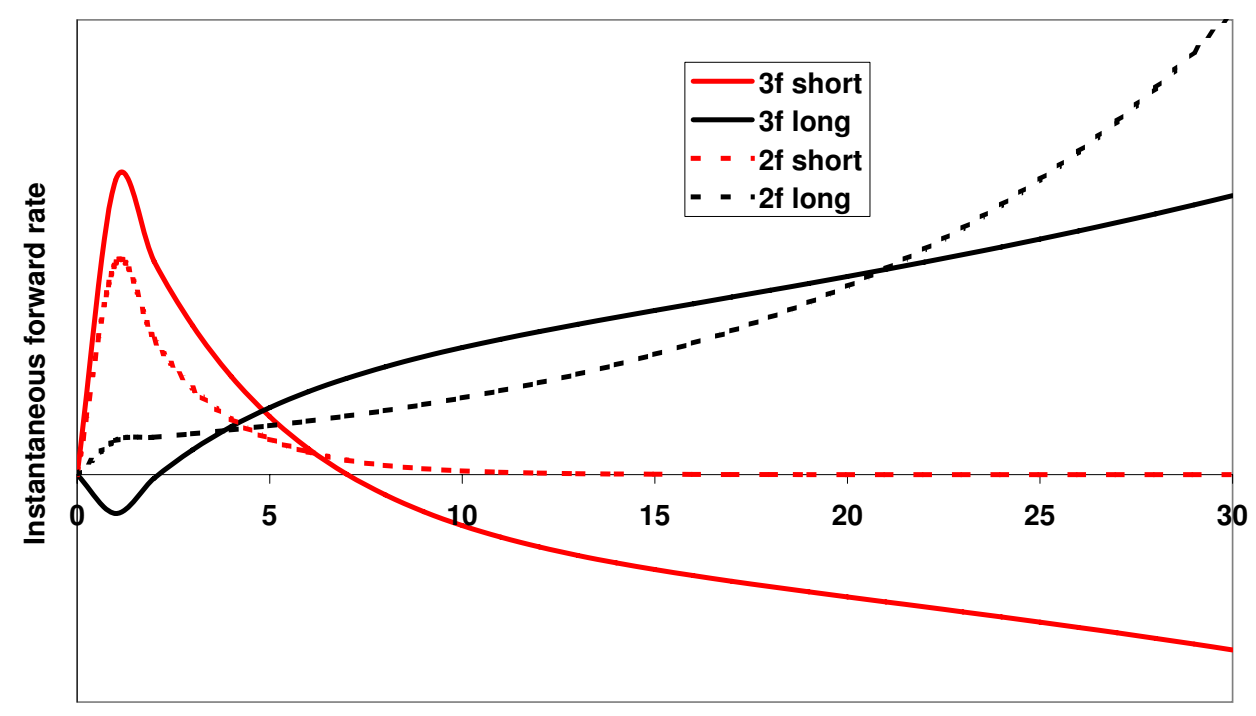

Maturity (years)

Figure 1: Effect of a decrease in demand on the term structure of instantaneous forward rates. The light (red) lines correspond to a "short-maturity" factor, concerning shocks at the five-year maturity, and the dark (black) lines to a "long-maturity" factor, concerning shocks at the twenty-year maturity. For each factor, the solid line represents the effect of a shock in the three-factor model, and the dashed line represents the same effect in a two-factor model where only that factor is present.

\footnotetext{
${ }^{26}$ When $a \approx \infty$, the system of $(K+1)^{2}$ non-linear equations in Proposition 10 reduces to $K$ equations, i.e., one equation per demand factor. The unknowns are the limit values of the scalars $\left\{\nu_{l}\right\}_{l=2, \ldots, K+1}$.

${ }^{27}$ The parameters $\left(\kappa_{r},\left\{\kappa_{\beta, k}\right\}_{k=1,2}, \sigma_{r},\left\{\sigma_{\beta, k}\right\}_{k=1,2}\right)$ do not matter when $a \approx \infty$.
} 
In the two-factor model, a decrease in demand raises all instantaneous forward rates, consistent with Corollary 3. Moreover, consistent with Proposition 6, the effect is stronger for short maturities in the case of $k_{s}$, and for long maturities in the case of $k_{\ell}$. In the three-factor model, a decrease in demand does not raise all forward rates - only those for maturities up to seven years in the case of $k_{s}$, and those for maturities of three years and longer in the case of $k_{\ell}$. Thus, the increase in forward rates is more local. The reason why some forward rates decrease has to do with the hedging activity of arbitrageurs. Following, for example, a decrease in demand for five-year bonds, arbitrageurs buy these bonds and hedge their long position by shorting bonds of other maturities. But while this hedge is effective when $k_{s}$ is the only factor, it ceases to be so when $k_{\ell}$ is added. This is because arbitrageurs are left exposed to an increase in demand for twenty-year bonds, which has its largest effect on long rates and therefore on arbitrageurs' short positions. To hedge that risk as well, arbitrageurs buy bonds of sufficiently long maturities, pushing down the corresponding forward rates. Figure 1 suggests that hedging becomes increasingly complicated as the number of factors increases. This, in turn, could imply that with a large number of factors, arbitrageurs become unwilling to integrate maturity markets and demand effects remain fully local. We leave an investigation of these issues for future work.

\section{Conclusion}

In this paper we build a preferred-habitat model of the term structure of interest rates. We assume that the term structure results from the interaction between investor maturity clienteles and riskaverse arbitrageurs. Because arbitrageurs are risk averse, shocks to clienteles' demand for bonds affect the term structure - and constitute an additional determinant of bond prices to current and expected future short rates. At the same time, because arbitrageurs render the term structure arbitrage-free, demand effects satisfy no-arbitrage restrictions and can be quite different from the underlying shocks. We show that the preferred-habitat view of the term structure generates a rich set of implications for bond risk premia, the effects of demand shocks and of shocks to short-rate expectations, the economic role of carry trades, and the transmission of monetary policy.

Our emphasis in this paper is mainly qualitative: use the closed-form solutions to understand key intuitions and comparative statics. We believe, however, that an equally rich set of implications can be derived on the quantitative front. Because of its affine structure, our model lends itself readily to structural estimation. Structural estimation can identify underlying parameters of the model such as the risk aversion of arbitrageurs and the demand shocks, which can then be linked and compared to observables. Moreover, the model so estimated can be a valuable policy tool, 
addressing what-if questions such as how purchases of bonds by the Central Bank, or issuance by the Treasury, can affect the term structure. The policy relevance of such questions has been underscored by recent events, e.g., the quantitative easing policies of Central Banks and the expansion of government debt. Our model is uniquely able to address such questions because of its focus on demand and supply shocks. Bringing the model to the data requires addressing a set of issues such as distinguishing between the real and the nominal term structure, introducing inflation, and determining the set of relevant risk factors. We believe that addressing these issues and performing the structural estimation are worthy of future work. 


\section{Appendix}

\section{A Proofs}

Proof of Lemma 1: Using (10), we can write (7) as

$$
d W_{t}=\left[W_{t} r_{t}+\int_{0}^{T} x_{t, \tau}\left(\mu_{t, \tau}-r_{t}\right) d \tau\right] d t-\left[\int_{0}^{T} x_{t, \tau} A_{r}(\tau) d \tau\right] \sigma_{r} d B_{r, t}
$$

and $(8)$ as

$$
\max _{\left\{x_{t, \tau}\right\}_{\tau \in(0, T]}}\left[\int_{0}^{T} x_{t, \tau}\left(\mu_{t, \tau}-r_{t}\right) d \tau-\frac{a \sigma_{r}^{2}}{2}\left[\int_{0}^{T} x_{t, \tau} A_{r}(\tau) d \tau\right]^{2}\right] .
$$

Point-wise maximization of (A.1) yields (12).

Proof of Proposition 1: Substituting $\mu_{t, \tau}$ from (11) and $\lambda_{r, t}$ from (14) into (12), we find

$$
\begin{aligned}
& A_{r}^{\prime}(\tau) r_{t}+C^{\prime}(\tau)-A_{r}(\tau) \kappa_{r}\left(\bar{r}-r_{t}\right)+\frac{1}{2} A_{r}(\tau)^{2} \sigma_{r}^{2}-r_{t} \\
& =A_{r}(\tau) a \sigma_{r}^{2} \int_{0}^{T} \alpha(\tau)\left[\bar{\beta} \tau-\left[A_{r}(\tau) r_{t}+C(\tau)\right]\right] A_{r}(\tau) d \tau
\end{aligned}
$$

This equation is affine in $r_{t}$. Setting linear terms in $r_{t}$ to zero yields (15), and setting constant terms to zero yields

$$
C^{\prime}(\tau)-\kappa_{r} \bar{r} A_{r}(\tau)+\frac{1}{2} \sigma_{r}^{2} A_{r}(\tau)^{2}=a \sigma_{r}^{2} A_{r}(\tau) \int_{0}^{T} \alpha(\tau)[\bar{\beta} \tau-C(\tau)] A_{r}(\tau) d \tau
$$

The differential equations (15) and (A.3) must be solved with the initial conditions $A_{r}(0)=C(0)=$ 0 . The solution to (15) is (16), provided that $\kappa_{r}^{*}$ is a solution to (18). Eq. (18) has a unique solution because $\int_{0}^{T} \alpha(\tau) A_{r}(\tau)^{2} d \tau$ is positive and decreasing in $\kappa_{r}^{*}$. The solution to (A.3) is

$$
C(\tau)=z_{r} \int_{0}^{\tau} A_{r}(u) d u-\frac{\sigma_{r}^{2}}{2} \int_{0}^{\tau} A_{r}(u)^{2} d u
$$

where

$$
z_{r} \equiv \kappa_{r} \bar{r}+a \sigma_{r}^{2} \int_{0}^{T} \alpha(\tau)[\bar{\beta} \tau-C(\tau)] A_{r}(\tau) d \tau
$$


Substituting $C(\tau)$ from (A.4) into (A.5), we find

$$
\begin{aligned}
z_{r}= & \kappa_{r} \bar{r}+a \sigma_{r}^{2} \bar{\beta} \int_{0}^{T} \alpha(\tau) \tau A_{r}(\tau) d \tau-a \sigma_{r}^{2} z_{r} \int_{0}^{T} \alpha(\tau)\left[\int_{0}^{\tau} A_{r}(u) d u\right] A_{r}(\tau) d \tau \\
& +\frac{a \sigma_{r}^{4}}{2} \int_{0}^{T} \alpha(\tau)\left[\int_{0}^{\tau} A_{r}(u)^{2} d u\right] A_{r}(\tau) d \tau \\
\Rightarrow z_{r} & =\frac{\kappa_{r} \bar{r}+a \sigma_{r}^{2} \bar{\beta} \int_{0}^{T} \alpha(\tau) \tau A_{r}(\tau) d \tau+\frac{a \sigma_{r}^{4}}{2} \int_{0}^{T} \alpha(\tau)\left[\int_{0}^{\tau} A_{r}(u)^{2} d u\right] A_{r}(\tau) d \tau}{1+a \sigma_{r}^{2} \int_{0}^{T} \alpha(\tau)\left[\int_{0}^{\tau} A_{r}(u) d u\right] A_{r}(\tau) d \tau}
\end{aligned}
$$

The function $C(\tau)$ coincides with (17) if $z_{r}=\kappa_{r}^{*} \bar{r}^{*}$. Eq. (19) implies that $z_{r}=\kappa_{r}^{*} \bar{r}^{*}$ if

$$
\frac{\kappa_{r}+a \sigma_{r}^{2} \int_{0}^{T} \alpha(\tau) \tau A_{r}(\tau) d \tau}{1+a \sigma_{r}^{2} \int_{0}^{T} \alpha(\tau)\left[\int_{0}^{\tau} A_{r}(u) d u\right] A_{r}(\tau) d \tau}=\kappa_{r}^{*}
$$

Eq. (A.6) follows from

$$
\begin{aligned}
& \kappa_{r}+a \sigma_{r}^{2} \int_{0}^{T} \alpha(\tau) \tau A_{r}(\tau) d \tau \\
& =\kappa_{r}^{*}+a \sigma_{r}^{2} \int_{0}^{T} \alpha(\tau)\left[\tau-A_{r}(\tau)\right] A_{r}(\tau) d \tau \\
& =\kappa_{r}^{*}+\kappa_{r}^{*} a \sigma_{r}^{2} \int_{0}^{T} \alpha(\tau)\left[\int_{0}^{\tau} A_{r}(u) d u\right] A_{r}(\tau) d \tau,
\end{aligned}
$$

where the first step follows from (18) and the second from (16).

To show that the risk-neutral dynamics are given by (20), we rewrite (A.2) as

$$
\begin{aligned}
& A_{r}^{\prime}(\tau) r_{t}+C^{\prime}(\tau)-A_{r}(\tau) \kappa_{r}\left(\bar{r}-r_{t}\right)+\frac{1}{2} A_{r}(\tau)^{2} \sigma_{r}^{2}-r_{t} \\
& =A_{r}(\tau) a \sigma_{r}^{2}\left[\int_{0}^{T} \alpha(\tau)[\bar{\beta} \tau-C(\tau)] A_{r}(\tau) d \tau-r_{t} \int_{0}^{T} \alpha(\tau) A_{r}(\tau)^{2} d \tau\right] \\
& \Leftrightarrow A_{r}^{\prime}(\tau) r_{t}+C^{\prime}(\tau)-A_{r}(\tau) \kappa_{r}\left(\bar{r}-r_{t}\right)+\frac{1}{2} A_{r}(\tau)^{2} \sigma_{r}^{2}-r_{t}=A_{r}(\tau)\left[z_{r}-\kappa_{r} \bar{r}-r_{t}\left(\kappa_{r}^{*}-\kappa_{r}\right)\right] \\
& \Leftrightarrow A_{r}^{\prime}(\tau) r_{t}+C^{\prime}(\tau)-A_{r}(\tau) \kappa_{r}^{*}\left(\bar{r}^{*}-r_{t}\right)+\frac{1}{2} A_{r}(\tau)^{2} \sigma_{r}^{2}-r_{t}=0,
\end{aligned}
$$

where the second step follows from (18) and (A.5), and the third from $z_{r}=\kappa_{r}^{*} \bar{r}^{*}$. The risk-neutral dynamics are given by (20) because of (A.7). 
Proof of Proposition 2: Eqs. (1), (2) and (9) imply that the dependent variable in (21) is

$$
\frac{1}{\Delta \tau}\left[A_{r}(\tau) r_{t}+C(\tau)-\left[A_{r}(\tau-\Delta \tau) r_{t+\Delta \tau}+C(\tau-\Delta \tau)\right]-\left[A_{r}(\Delta \tau) r_{t}+C(\Delta \tau)\right]\right]
$$

and the independent variable is

$$
\frac{1}{\Delta \tau}\left[A_{r}(\tau) r_{t}+C(\tau)-\left[A_{r}(\tau-\Delta \tau) r_{t}+C(\tau-\Delta \tau)\right]-\left[A_{r}(\Delta \tau) r_{t}+C(\Delta \tau)\right]\right]
$$

Therefore, the regression coefficient in (21) is

$$
\gamma_{\mathrm{FB}}=\frac{\operatorname{Cov}\left[\left[A_{r}(\tau)-A_{r}(\Delta \tau)\right] r_{t}-A_{r}(\tau-\Delta \tau) r_{t+\Delta \tau},\left[A_{r}(\tau)-A_{r}(\tau-\Delta \tau)-A_{r}(\Delta \tau)\right] r_{t}\right]}{\operatorname{Var}\left[\left[A_{r}(\tau)-A_{r}(\tau-\Delta \tau)-A_{r}(\Delta \tau)\right] r_{t}\right]}
$$

Eq. (3) implies that

$$
E_{t}\left(r_{t+\tau}\right)=\left(1-e^{-\kappa_{r} \tau}\right) \bar{r}+e^{-\kappa_{r} \tau} r_{t}
$$

which, in turn, implies that

$$
\operatorname{Cov}\left(r_{t+\Delta \tau}, r_{t}\right)=\operatorname{Var}\left(r_{t}\right) e^{-\kappa_{r} \Delta \tau}
$$

Substituting into (A.8), we find

$$
\gamma_{\mathrm{FB}}=\frac{A_{r}(\tau)-A_{r}(\tau-\Delta \tau) e^{-\kappa_{r} \Delta \tau}-A_{r}(\Delta \tau)}{A_{r}(\tau)-A_{r}(\tau-\Delta \tau)-A_{r}(\Delta \tau)}
$$

Taking the limit $\Delta \tau \rightarrow 0$ and noting from (16) that $A_{r}(\Delta \tau) / \Delta \tau \rightarrow 1$, we find

$$
\gamma_{\mathrm{FB}} \rightarrow \frac{A_{r}^{\prime}(\tau)+\kappa_{r} A_{r}(\tau)-1}{A_{r}^{\prime}(\tau)-1}=\frac{\left(\kappa_{r}^{*}-\kappa_{r}\right) A_{r}(\tau)}{\kappa_{r}^{*} A_{r}(\tau)}=\frac{\kappa_{r}^{*}-\kappa_{r}}{\kappa_{r}^{*}}
$$

where the second step follows from (15). The proposition follows from (A.10) and $\kappa_{r}^{*}>\kappa_{r}$.

Proof of Proposition 3: Eq. (22) implies that

$$
\frac{\partial f_{t, \tau}}{\partial r_{t}}=A_{r}^{\prime}(\tau)=e^{-\kappa_{r}^{*} \tau}
$$

where the second step follows from (16). Eq. (A.9) implies that

$$
\frac{\partial E_{t}\left(r_{t+\tau}\right)}{\partial r_{t}}=e^{-\kappa_{r} \tau}
$$


The proposition follows from (A.11), (A.12) and $\kappa_{r}^{*}>\kappa_{r}$.

Proof of Corollary 1: The key observation is that the mean-reversion rate $\kappa_{r}^{*}$ under the riskneutral measure is an increasing function of $a$. Indeed, $\kappa_{r}^{*}$ is defined implicitly by (18), whose left-hand side is increasing in $\kappa_{r}^{*}$, and right-hand side is decreasing in $\kappa_{r}^{*}$ and increasing in $a$. The first claim in the corollary follows from this observation and (A.10). The second claim follows from this observation and (A.11).

Proof of Lemma 2: Using (24), we can write (7) as

$$
d W_{t}=\left[W_{t} r_{t}+\int_{0}^{T} x_{t, \tau}\left(\mu_{t, \tau}-r_{t}\right) d \tau\right] d t-\left[\int_{0}^{T} x_{t, \tau} A_{r}(\tau) d \tau\right] \sigma_{r} d B_{r, t}-\left[\int_{0}^{T} x_{t, \tau} A_{\beta}(\tau) d \tau\right] \sigma_{\beta} d B_{\beta, t}
$$

and (8) as

$$
\max _{\left\{x_{t, \tau}\right\}_{\tau \in(0, T]}}\left[\int_{0}^{T} x_{t, \tau}\left(\mu_{t, \tau}-r_{t}\right) d \tau-\frac{a \sigma_{r}^{2}}{2}\left[\int_{0}^{T} x_{t, \tau} A_{r}(\tau) d \tau\right]^{2}-\frac{a \sigma_{\beta}^{2}}{2}\left[\int_{0}^{T} x_{t, \tau} A_{\beta}(\tau) d \tau\right]^{2}\right] .
$$

Point-wise maximization of (A.13) yields (26).

Proof of Proposition 4: Setting $x_{t, \tau}=-y_{t, \tau}$ in (27) and (28), and using (1), (4), (5), (23) and $K=1$, we find

$$
\begin{aligned}
& \lambda_{r, t}=a \sigma_{r}^{2} \int_{0}^{T} \alpha(\tau)\left[\left[\bar{\beta}+\theta(\tau) \beta_{t}\right] \tau-\left[A_{r}(\tau) r_{t}+A_{\beta}(\tau) \beta_{t}+C(\tau)\right]\right] A_{r}(\tau) d \tau \\
& \lambda_{\beta, t}=a \sigma_{\beta}^{2} \int_{0}^{T} \alpha(\tau)\left[\left[\bar{\beta}+\theta(\tau) \beta_{t}\right] \tau-\left[A_{r}(\tau) r_{t}+A_{\beta}(\tau) \beta_{t}+C(\tau)\right]\right] A_{\beta}(\tau) d \tau
\end{aligned}
$$

Substituting $\mu_{t, \tau}$ from (25), $\lambda_{r, t}$ from (A.14) and $\lambda_{\beta, t}$ from (A.15) into (26), we find

$$
\begin{aligned}
& A_{r}^{\prime}(\tau) r_{t}+A_{\beta}(\tau) \beta_{t}+C^{\prime}(\tau)-A_{r}(\tau) \kappa_{r}\left(\bar{r}-r_{t}\right)+A_{\beta}(\tau) \kappa_{\beta} \beta_{t}+\frac{1}{2} A_{r}(\tau)^{2} \sigma_{r}^{2}+\frac{1}{2} A_{\beta}(\tau)^{2} \sigma_{\beta}^{2}-r_{t} \\
& =A_{r}(\tau) a \sigma_{r}^{2} \int_{0}^{T} \alpha(\tau)\left[\left[\bar{\beta}+\theta(\tau) \beta_{t}\right] \tau-\left[A_{r}(\tau) r_{t}+A_{\beta}(\tau) \beta_{t}+C(\tau)\right]\right] A_{r}(\tau) d \tau \\
& +A_{\beta}(\tau) a \sigma_{\beta}^{2} \int_{0}^{T} \alpha(\tau)\left[\left[\bar{\beta}+\theta(\tau) \beta_{t}\right] \tau-\left[A_{r}(\tau) r_{t}+A_{\beta}(\tau) \beta_{t}+C(\tau)\right]\right] A_{\beta}(\tau) d \tau
\end{aligned}
$$


This equation is affine in $\left(r_{t}, \beta_{t}\right)$. Setting linear terms in $\left(r_{t}, \beta_{t}\right)$ to zero yields (29) and (30), and setting constant terms to zero yields

$$
\begin{aligned}
& C^{\prime}(\tau)-\kappa_{r} \bar{r} A_{r}(\tau)+\frac{1}{2} \sigma_{r}^{2} A_{r}(\tau)^{2}+\frac{1}{2} \sigma_{\beta}^{2} A_{\beta}(\tau)^{2} \\
& =a \sigma_{r}^{2} A_{r}(\tau) \int_{0}^{T} \alpha(\tau)[\bar{\beta} \tau-C(\tau)] A_{r}(\tau) d \tau+a \sigma_{\beta}^{2} A_{\beta}(\tau) \int_{0}^{T} \alpha(\tau)[\bar{\beta} \tau-C(\tau)] A_{\beta}(\tau) d \tau
\end{aligned}
$$

The differential equations (29), (30) and (A.17) must be solved with the initial conditions $A_{r}(0)=$ $A_{\beta}(0)=C(0)=0$.

Using the diagonalization

$$
\left[\begin{array}{cc}
\kappa_{r} & 0 \\
0 & \kappa_{\beta}
\end{array}\right]-M \equiv P^{-1}\left[\begin{array}{cc}
\nu_{1} & 0 \\
0 & \nu_{2}
\end{array}\right] P
$$

and can write the system of (29) and (30) as

$$
P\left[\begin{array}{l}
A_{r}^{\prime}(\tau) \\
A_{\beta}^{\prime}(\tau)
\end{array}\right]+\left[\begin{array}{cc}
\nu_{1} & 0 \\
0 & \nu_{2}
\end{array}\right] P\left[\begin{array}{c}
A_{r}(\tau) \\
A_{\beta}(\tau)
\end{array}\right]-P\left[\begin{array}{l}
1 \\
0
\end{array}\right]=0
$$

The solution to (A.18) with the initial condition $A_{r}(0)=A_{\beta}(0)=0$ is

$$
\begin{aligned}
& P\left[\begin{array}{l}
A_{r}(\tau) \\
A_{\beta}(\tau)
\end{array}\right]=\left[\begin{array}{cc}
\frac{1-e^{-\nu_{1} \tau}}{\nu_{1}} & 0 \\
0 & \frac{1-e^{-\nu_{2} \tau}}{\nu_{2}}
\end{array}\right] P\left[\begin{array}{l}
1 \\
0
\end{array}\right] \\
& \Rightarrow\left[\begin{array}{l}
A_{r}(\tau) \\
A_{\beta}(\tau)
\end{array}\right]=\frac{1-e^{-\nu_{1} \tau}}{\nu_{1}}\left[\begin{array}{l}
1 \\
0
\end{array}\right]+\left(\frac{1-e^{-\nu_{2} \tau}}{\nu_{2}}-\frac{1-e^{-\nu_{1} \tau}}{\nu_{1}}\right) P^{-1}\left[\begin{array}{ll}
0 & 0 \\
0 & 1
\end{array}\right] P\left[\begin{array}{l}
1 \\
0
\end{array}\right],
\end{aligned}
$$

and has the form in the proposition. To determine the scalars $\left(\gamma_{r}, \gamma_{\beta}, \nu_{1}, \nu_{2}\right)$, we substitute (32) and (33) into (29) and (30), and identify terms in $\frac{1-e^{-\nu_{1} \tau}}{\nu_{1}}$ and $\frac{1-e^{-\nu_{2} \tau}}{\nu_{2}}$. This yields

$$
\begin{aligned}
& \left(1-\gamma_{r}\right)\left(\nu_{1}-\kappa_{r}+M_{1,1}\right)-\gamma_{\beta} M_{1,2}=0, \\
& \gamma_{r}\left(\nu_{2}-\kappa_{r}+M_{1,1}\right)+\gamma_{\beta} M_{1,2}=0,
\end{aligned}
$$

in the case of (29) and

$$
\begin{aligned}
& \gamma_{\beta}\left(\nu_{1}-\kappa_{\beta}+M_{2,2}\right)-\left(1-\gamma_{r}\right) M_{2,1}=0, \\
& -\gamma_{\beta}\left(\nu_{2}-\kappa_{\beta}+M_{2,2}\right)-\gamma_{r} M_{2,1}=0
\end{aligned}
$$


in the case of (30). Combining (A.19) and (A.20), we find the equivalent equations

$$
\begin{aligned}
& \nu_{1}+\gamma_{r}\left(\nu_{2}-\nu_{1}\right)-\kappa_{r}+M_{1,1}=0, \\
& \gamma_{r}\left(1-\gamma_{r}\right)\left(\nu_{1}-\nu_{2}\right)-\gamma_{\beta} M_{1,2}=0,
\end{aligned}
$$

and combining (A.21) and (A.22), we find the equivalent equations

$$
\begin{aligned}
& \gamma_{\beta}\left(\nu_{1}-\nu_{2}\right)-M_{2,1}=0, \\
& \kappa_{\beta}-\nu_{2}-\gamma_{r}\left(\nu_{1}-\nu_{2}\right)-M_{2,2}=0 .
\end{aligned}
$$

Setting

$$
\begin{aligned}
& F\left(\nu, \nu^{\prime}\right) \equiv \int_{0}^{T} \alpha(\tau) \frac{1-e^{-\nu \tau}}{\nu} \frac{1-e^{-\nu^{\prime} \tau}}{\nu^{\prime}} d \tau, \\
& \hat{F}\left(\nu, \nu^{\prime}\right) \equiv F\left(\nu, \nu^{\prime}\right)-F(\nu, \nu), \\
& \hat{\hat{F}}\left(\nu, \nu^{\prime}\right) \equiv F(\nu, \nu)+F\left(\nu^{\prime}, \nu^{\prime}\right)-2 F\left(\nu, \nu^{\prime}\right), \\
& G(\nu) \equiv \int_{0}^{T} \alpha(\tau) \tau \theta(\tau) \frac{1-e^{-\nu \tau}}{\nu} d \tau, \\
& \hat{G}\left(\nu, \nu^{\prime}\right) \equiv G\left(\nu^{\prime}\right)-G(\nu),
\end{aligned}
$$

and using (31)-(33), we can write the system of (A.23)-(A.26) as

$$
\begin{aligned}
& \nu_{1}+\gamma_{r}\left(\nu_{2}-\nu_{1}\right)-\kappa_{r}-a \sigma_{r}^{2}\left[F\left(\nu_{1}, \nu_{1}\right)+2 \gamma_{r} \hat{F}\left(\nu_{1}, \nu_{2}\right)+\gamma_{r}^{2} \hat{\hat{F}}\left(\nu_{1}, \nu_{2}\right)\right]=0, \\
& \gamma_{r}\left(1-\gamma_{r}\right)\left(\nu_{1}-\nu_{2}\right)+a \sigma_{\beta}^{2} \gamma_{\beta}^{2}\left[\hat{F}\left(\nu_{1}, \nu_{2}\right)+\gamma_{r} \hat{\hat{F}}\left(\nu_{1}, \nu_{2}\right)\right]=0, \\
& \gamma_{\beta}\left(\nu_{1}-\nu_{2}\right)-a \sigma_{r}^{2}\left[G\left(\nu_{1}\right)+\gamma_{r} \hat{G}\left(\nu_{1}, \nu_{2}\right)-\gamma_{\beta}\left[\hat{F}\left(\nu_{1}, \nu_{2}\right)+\gamma_{r} \hat{\hat{F}}\left(\nu_{1}, \nu_{2}\right)\right]\right]=0, \\
& \kappa_{\beta}-\nu_{2}-\gamma_{r}\left(\nu_{1}-\nu_{2}\right)-a \sigma_{\beta}^{2} \gamma_{\beta}\left[\hat{G}\left(\nu_{1}, \nu_{2}\right)-\gamma_{\beta} \hat{\hat{F}}\left(\nu_{1}, \nu_{2}\right)\right]=0,
\end{aligned}
$$

i.e., as a system of four scalar non-linear equations in the unknowns $\left(\nu_{1}, \nu_{2}, \gamma_{r}, \gamma_{\beta}\right)$.

The solution to (A.17) is

$$
C(\tau)=z_{r} \int_{0}^{\tau} A_{r}(u) d u+z_{\beta} \int_{0}^{\tau} A_{\beta}(u) d u-\frac{\sigma_{r}^{2}}{2} \int_{0}^{\tau} A_{r}(u)^{2} d u-\frac{\sigma_{\beta}^{2}}{2} \int_{0}^{\tau} A_{\beta}(u)^{2} d u
$$


where

$$
\begin{aligned}
& z_{r} \equiv \kappa_{r} \bar{r}+a \sigma_{r}^{2} \int_{0}^{T} \alpha(\tau)[\bar{\beta} \tau-C(\tau)] A_{r}(\tau) d \tau, \\
& z_{\beta} \equiv a \sigma_{\beta}^{2} \int_{0}^{T} \alpha(\tau)[\bar{\beta} \tau-C(\tau)] A_{\beta}(\tau) d \tau .
\end{aligned}
$$

Substituting $C(\tau)$ from (A.31) into (A.32) and (A.33), we can derive $\left(z_{r}, z_{\beta}\right)$ as the solution to a linear system of equations.

Proof of Proposition 5: Consider first the case $a \approx 0$. Setting $\left(\gamma_{r}, \gamma_{\beta}\right)=\left(a^{3} c_{r}, a c_{\beta}\right)$, we can write (A.27)-(A.30) as

$$
\begin{aligned}
& \nu_{1}+a^{3} c_{r}\left(\nu_{2}-\nu_{1}\right)-\kappa_{r}-a \sigma_{r}^{2}\left[F\left(\nu_{1}, \nu_{1}\right)+2 a^{3} c_{r} \hat{F}\left(\nu_{1}, \nu_{2}\right)+a^{6} c_{r}^{2} \hat{\hat{F}}\left(\nu_{1}, \nu_{2}\right)\right]=0, \\
& c_{r}\left(1-a^{3} c_{r}\right)\left(\nu_{1}-\nu_{2}\right)+\sigma_{\beta}^{2} c_{\beta}^{2}\left[\hat{F}\left(\nu_{1}, \nu_{2}\right)+a^{3} c_{r} \hat{\hat{F}}\left(\nu_{1}, \nu_{2}\right)\right]=0, \\
& c_{\beta}\left(\nu_{1}-\nu_{2}\right)-\sigma_{r}^{2}\left[G\left(\nu_{1}\right)+a^{3} c_{r} \hat{G}\left(\nu_{1}, \nu_{2}\right)-a c_{\beta}\left[\hat{F}\left(\nu_{1}, \nu_{2}\right)+a^{3} c_{r} \hat{\hat{F}}\left(\nu_{1}, \nu_{2}\right)\right]\right]=0, \\
& \kappa_{\beta}-\nu_{2}-a^{3} c_{r}\left(\nu_{1}-\nu_{2}\right)-a^{2} \sigma_{\beta}^{2} c_{\beta}\left[\hat{G}\left(\nu_{1}, \nu_{2}\right)-a c_{\beta} \hat{\hat{F}}\left(\nu_{1}, \nu_{2}\right)\right]=0 .
\end{aligned}
$$

The asymptotic behavior of $\left(\nu_{1}, \nu_{2}, \gamma_{r}, \gamma_{\beta}\right)$ is as in the proposition if (A.34)-(A.37) has a non-zero solution $\left(\nu_{1}, \nu_{2}, c_{r}, c_{\beta}\right)$ for $a=0$. For $a=0$, (A.34) implies that $\nu_{1}=\kappa_{r}$, (A.37) implies that $\nu_{2}=\kappa_{\beta},($ A.36) implies that

$$
c_{\beta}=\underline{c}_{\beta} \equiv \frac{\sigma_{r}^{2} G\left(\kappa_{r}\right)}{\kappa_{r}-\kappa_{\beta}}
$$

and (A.35) implies that

$$
c_{r}=\underline{c}_{r} \equiv-\frac{\sigma_{\beta}^{2} \underline{c}_{\beta}^{2} \hat{F}\left(\kappa_{r}, \kappa_{\beta}\right)}{\kappa_{r}-\kappa_{\beta}} .
$$

Consider next the case $a \approx \infty$. Setting $\left(\nu_{1}, \gamma_{r}\right)=\left(a^{\frac{1}{3}} n_{1}, a^{-\frac{1}{3}} c_{r}\right)$, we can write (A.27)-(A.30) as

$$
\begin{aligned}
n_{1}+a^{-\frac{2}{3}} c_{r}\left(\nu_{2}-a^{\frac{1}{3}} n_{1}\right)-a^{-\frac{1}{3}} \kappa_{r} & -a^{\frac{2}{3}} \sigma_{r}^{2}\left[F\left(a^{\frac{1}{3}} n_{1}, a^{\frac{1}{3}} n_{1}\right)\right. \\
& \left.+2 a^{-\frac{1}{3}} c_{r} \hat{F}\left(a^{\frac{1}{3}} n_{1}, \nu_{2}\right)+a^{-\frac{2}{3}} c_{r}^{2} \hat{\hat{F}}\left(a^{\frac{1}{3}} n_{1}, \nu_{2}\right)\right]=0,
\end{aligned}
$$




$$
\begin{gathered}
a^{-1} c_{r}\left(1-a^{-\frac{1}{3}} c_{r}\right)\left(a^{\frac{1}{3}} n_{1}-\nu_{2}\right)+a^{\frac{1}{3}} \sigma_{\beta}^{2} \gamma_{\beta}^{2}\left[\hat{F}\left(a^{\frac{1}{3}} n_{1}, \nu_{2}\right)+a^{-\frac{1}{3}} c_{r} \hat{\hat{F}}\left(a^{\frac{1}{3}} n_{1}, \nu_{2}\right)\right]=0, \\
a^{-\frac{2}{3}} \gamma_{\beta}\left(a^{\frac{1}{3}} n_{1}-\nu_{2}\right)-a^{\frac{1}{3}} \sigma_{r}^{2}\left[G\left(a^{\frac{1}{3}} n_{1}\right)+a^{-\frac{1}{3}} c_{r} \hat{G}\left(a^{\frac{1}{3}} n_{1}, \nu_{2}\right)\right. \\
\left.-\gamma_{\beta}\left[\hat{F}\left(a^{\frac{1}{3}} n_{1}, \nu_{2}\right)+a^{-\frac{1}{3}} c_{r} \hat{\hat{F}}\left(a^{\frac{1}{3}} n_{1}, \nu_{2}\right)\right]\right]=0, \\
a^{-1}\left[\kappa_{\beta}-\nu_{2}-a^{-\frac{1}{3}} c_{r}\left(a^{\frac{1}{3}} n_{1}-\nu_{2}\right)\right]-\sigma_{\beta}^{2} \gamma_{\beta}\left[\hat{G}\left(a^{\frac{1}{3}} n_{1}, \nu_{2}\right)-\gamma_{\beta} \hat{\hat{F}}\left(a^{\frac{1}{3}} n_{1}, \nu_{2}\right)\right]=0 .
\end{gathered}
$$

The asymptotic behavior of $\left(\nu_{1}, \nu_{2}, \gamma_{r}, \gamma_{\beta}\right)$ is as in the proposition if (A.40)-(A.43) has a non-zero solution $\left(n_{1}, \nu_{2}, c_{r}, \gamma_{\beta}\right)$ for $a=\infty$. Noting that

$$
\begin{aligned}
& \lim _{a \rightarrow \infty} a^{\frac{2}{3}} F\left(a^{\frac{1}{3}} n_{1}, a^{\frac{1}{3}} n_{1}\right)=\frac{1}{n_{1}^{2}} \int_{0}^{T} \alpha(\tau) d \tau, \\
& \lim _{a \rightarrow \infty} a^{\frac{1}{3}} F\left(a^{\frac{1}{3}} n_{1}, \nu_{2}\right)=\frac{1}{n_{1}} \int_{0}^{T} \alpha(\tau) \frac{1-e^{-\nu_{2} \tau}}{\nu_{2}} d \tau, \\
& \lim _{a \rightarrow \infty} a^{\frac{1}{3}} G\left(a^{\frac{1}{3}} n_{1}\right)=\frac{1}{n_{1}} \int_{0}^{T} \alpha(\tau) \tau \theta(\tau) d \tau,
\end{aligned}
$$

we can write (A.40)-(A.43) for $a=\infty$ as

$$
\begin{aligned}
& n_{1}-\sigma_{r}^{2}\left[\frac{1}{n_{1}^{2}} \int_{0}^{T} \alpha(\tau) d \tau+2 c_{r} \frac{1}{n_{1}} \int_{0}^{T} \alpha(\tau) \frac{1-e^{-\nu_{2} \tau}}{\nu_{2}} d \tau+c_{r}^{2} \int_{0}^{T} \alpha(\tau)\left(\frac{1-e^{-\nu_{2} \tau}}{\nu_{2}}\right)^{2} d \tau\right]=0, \\
& \frac{1}{n_{1}} \int_{0}^{T} \alpha(\tau) \frac{1-e^{-\nu_{2} \tau}}{\nu_{2}} d \tau+c_{r} \int_{0}^{T} \alpha(\tau)\left(\frac{1-e^{-\nu_{2} \tau}}{\nu_{2}}\right)^{2} d \tau=0 \\
& \frac{1}{n_{1}} \int_{0}^{T} \alpha(\tau) \tau \theta(\tau) d \tau+c_{r} \int_{0}^{T} \alpha(\tau) \tau \theta(\tau) \frac{1-e^{-\nu_{2} \tau}}{\nu_{2}} d \tau \\
& -\gamma_{\beta}\left[\frac{1}{n_{1}} \int_{0}^{T} \alpha(\tau) \frac{1-e^{-\nu_{2} \tau}}{\nu_{2}} d \tau+c_{r} \int_{0}^{T} \alpha(\tau)\left(\frac{1-e^{-\nu_{2} \tau}}{\nu_{2}}\right)^{2} d \tau\right]=0, \\
& \int_{0}^{T} \alpha(\tau) \tau \theta(\tau) \frac{1-e^{-\nu_{2} \tau}}{\nu_{2}} d \tau-\gamma_{\beta} \int_{0}^{T} \alpha(\tau)\left(\frac{1-e^{-\nu_{2} \tau}}{\nu_{2}}\right)^{2} d \tau=0 .
\end{aligned}
$$

Using (A.45) to eliminate $c_{r}$ in (A.46), we find the following equation for $\nu_{2}$ :

$$
\frac{\int_{0}^{T} \alpha(\tau) \tau \theta(\tau) \frac{1-e^{-\nu_{2} \tau}}{\nu_{2}} d \tau}{\int_{0}^{T} \alpha(\tau) \tau \theta(\tau) d \tau}=\frac{\int_{0}^{T} \alpha(\tau)\left(\frac{1-e^{-\nu_{2} \tau}}{\nu_{2}}\right)^{2} d \tau}{\int_{0}^{T} \alpha(\tau) \frac{1-e^{-\nu_{2} \tau}}{\nu_{2}} d \tau}
$$


Eq. (A.48) has a solution $\bar{\nu}_{2}$. Indeed, when $\nu_{2}$ goes to $\infty$, the left-hand side is

$$
\frac{1}{\nu_{2}}\left[1-\frac{\int_{0}^{T} \alpha(\tau) \tau \theta(\tau) e^{-\nu_{2} \tau} d \tau}{\int_{0}^{T} \alpha(\tau) \tau \theta(\tau) d \tau}\right]=\frac{1}{\nu_{2}}\left[1+o\left(\frac{1}{\nu_{2}}\right)\right]
$$

and exceeds the right-hand side, which is

$$
\frac{1}{\nu_{2}}\left[1-\frac{\int_{0}^{T} \alpha(\tau)\left(1-e^{-\nu_{2} \tau}\right) e^{-\nu_{2} \tau} d \tau}{\int_{0}^{T} \alpha(\tau)\left(1-e^{-\nu_{2} \tau}\right) d \tau}\right]=\frac{1}{\nu_{2}}\left[1-\frac{\alpha(0)}{\nu_{2} \int_{0}^{T} \alpha(\tau) d \tau}+o\left(\frac{1}{\nu_{2}}\right)\right] .
$$

When instead $\nu_{2}$ goes to $-\infty$, the left-hand side is

$$
\frac{e^{-\nu_{2} T}}{\nu_{2}} \frac{\int_{0}^{T} \alpha(\tau) \tau \theta(\tau)\left[e^{\nu_{2} T}-e^{\nu_{2}(T-\tau)}\right] d \tau}{\int_{0}^{T} \alpha(\tau) \tau \theta(\tau) d \tau}=\frac{e^{-\nu_{2} T}}{\nu_{2}^{2}} \frac{\alpha(T) T \theta(T)}{\int_{0}^{T} \alpha(\tau) \tau \theta(\tau) d \tau}+o\left(\frac{1}{\nu_{2}^{2}}\right)
$$

and is smaller than the right-hand side, which is

$$
\frac{e^{-\nu_{2} T}}{\nu_{2}} \frac{\int_{0}^{T} \alpha(\tau)\left[e^{\nu_{2} T}-e^{\nu_{2}(T-\tau)}\right]^{2} d \tau}{\int_{0}^{T} \alpha(\tau)\left[e^{\nu_{2} T}-e^{\nu_{2}(T-\tau)}\right] d \tau}=\frac{e^{-\nu_{2} T}}{-2 \nu_{2}}+o\left(\frac{1}{\nu_{2}}\right)
$$

Using (A.45) to eliminate $c_{r}$ in (A.44), we find

$$
n_{1}=\bar{n}_{1} \equiv \sigma_{r}^{\frac{2}{3}}\left[\int_{0}^{T} \alpha(\tau) d \tau-\frac{\left[\int_{0}^{T} \alpha(\tau) \frac{1-e^{-\bar{\nu}_{2} \tau}}{\bar{\nu}_{2}} d \tau\right]^{2}}{\int_{0}^{T} \alpha(\tau)\left(\frac{1-e^{-\bar{\nu}_{2} \tau}}{\bar{\nu}_{2}}\right)^{2} d \tau}\right]^{\frac{1}{3}}
$$

Eq. (A.45) implies that

$$
c_{r}=\bar{c}_{r} \equiv-\frac{1}{\bar{n}_{1}} \frac{\int_{0}^{T} \alpha(\tau) \frac{1-e^{-\bar{\nu}_{2} \tau}}{\bar{\nu}_{2}} d \tau}{\int_{0}^{T} \alpha(\tau)\left(\frac{1-e^{-\bar{\nu}_{2} \tau}}{\bar{\nu}_{2}}\right)^{2} d \tau},
$$

and (A.47) implies that

$$
\gamma_{\beta}=\bar{\gamma}_{\beta} \equiv \frac{\int_{0}^{T} \alpha(\tau) \tau \theta(\tau) \frac{1-e^{-\bar{\nu}_{2} \tau}}{\bar{\nu}_{2}} d \tau}{\int_{0}^{T} \alpha(\tau)\left(\frac{1-e^{-\bar{\nu}_{2} \tau}}{\bar{\nu}_{2}}\right)^{2} d \tau}
$$

Eq. (A.49) implies that $\bar{n}_{1}>0$ because of the Cauchy-Schwarz inequality. Eq. (A.50) implies that $\bar{c}_{r}<0$. 
Proof of Corollary 2: Proposition 4 implies that the shock's effect on instantaneous forward rates is

$$
A_{r}^{\prime}(\tau)=e^{-\nu_{1} \tau}\left(1-\gamma_{r}\right)\left[1+\frac{\gamma_{r}}{1-\gamma_{r}} e^{\left(\nu_{1}-\nu_{2}\right) \tau}\right]
$$

When $a \approx \infty$, the term outside the square brackets is positive. Moreover, the term inside the square brackets is positive for $\tau<\tau_{r}$ and negative for $\tau>\tau_{r}$, where

$$
\tau_{r} \equiv \frac{\log \left(-\frac{1-\gamma_{r}}{\gamma_{r}}\right)}{\nu_{1}-\nu_{2}} \approx \frac{\log \left(-\frac{a^{\frac{1}{3}}}{\bar{c}_{r}}\right)}{a^{\frac{1}{3}} \bar{n}_{1}}
$$

Since $\tau_{r} \approx 0, \tau_{r} \in[0, T]$.

The shock's effect on spot rates is $A_{r}(\tau) / \tau$ and has the same sign as $A_{r}(\tau)$. Since $A_{r}^{\prime}(\tau)$ is positive and then negative, $A_{r}(\tau)=\int_{0}^{T} A_{r}^{\prime}(\tau) d \tau$ is positive, or positive and then negative. Propositions 4 and 5 imply that when $a \approx \infty$,

$$
A_{r}(T) \approx a^{-\frac{1}{3}}\left(\frac{1}{\bar{n}_{1}}+\bar{c}_{r} \frac{1-e^{-\bar{\nu}_{2} T}}{\bar{\nu}_{2}}\right)=\frac{a^{-\frac{1}{3}} \int_{0}^{T} \alpha(\tau)\left(\frac{1-e^{-\bar{\nu}_{2} \tau}}{\bar{\nu}_{2}}\right)\left(\frac{1-e^{-\bar{\nu}_{2} \tau}}{\bar{\nu}_{2}}-\frac{1-e^{-\bar{\nu}_{2} T}}{\bar{\nu}_{2}}\right) d \tau}{\bar{n}_{1} \int_{0}^{T} \alpha(\tau)\left(\frac{1-e^{-\bar{\nu}_{2} \tau}}{\bar{\nu}_{2}}\right)^{2} d \tau}<0
$$

where the second step follows from (A.50). Since $A_{r}(T)<0, A_{r}(\tau)$ is positive and then negative.

Proof of Proposition 6: We first show that $\bar{\nu}_{2}$ is decreasing in $x$. Recall that $\bar{\nu}_{2}$ solves (A.48), which can be written as

$$
\frac{\int_{0}^{T} \alpha(\tau) \tau \theta(\tau, x) \frac{1-e^{-\nu_{2} \tau}}{\nu_{2}} d \tau}{\int_{0}^{T} \alpha(\tau) \tau \theta(\tau, x) d \tau}-\frac{\int_{0}^{T} \alpha(\tau)\left(\frac{1-e^{-\nu_{2} \tau}}{\nu_{2}}\right)^{2} d \tau}{\int_{0}^{T} \alpha(\tau) \frac{1-e^{-\nu_{2} \tau}}{\nu_{2}} d \tau}=0
$$

and where the left-hand side is negative when $\nu_{2}$ goes to $-\infty$ and positive when $\nu_{2}$ goes to $\infty$. The solution to (A.52) is decreasing in $x$ if the left-hand side of (A.52) is increasing in $x$. The derivative 
with respect to $x$ has the same sign as

$$
\begin{aligned}
& \int_{0}^{T} \alpha(\tau) \tau \theta_{x}(\tau, x) \frac{1-e^{-\nu_{2} \tau}}{\nu_{2}} d \tau \int_{0}^{T} \alpha(\tau) \tau \theta(\tau, x) d \tau-\int_{0}^{T} \alpha(\tau) \tau \theta(\tau, x) \frac{1-e^{-\nu_{2} \tau}}{\nu_{2}} d \tau \int_{0}^{T} \alpha(\tau) \tau \theta_{x}(\tau, x) d \tau \\
& =\int_{[0, T]^{2}} \phi\left(\tau_{1}\right) \phi\left(\tau_{2}\right) \frac{\theta_{x}\left(\tau_{1}, x\right)}{\theta\left(\tau_{1}, x\right)} f\left(\tau_{1}\right) d \tau_{1} d \tau_{2}-\int_{[0, T]^{2}} \phi\left(\tau_{1}\right) \phi\left(\tau_{2}\right) \frac{\theta_{x}\left(\tau_{1}, x\right)}{\theta\left(\tau_{1}, x\right)} f\left(\tau_{2}\right) d \tau_{1} d \tau_{2} \\
& =\int_{0 \leq \tau_{1} \leq \tau_{2} \leq T} \phi\left(\tau_{1}\right) \phi\left(\tau_{2}\right)\left[\frac{\theta_{x}\left(\tau_{1}, x\right)}{\theta\left(\tau_{1}, x\right)} f\left(\tau_{1}\right)+\frac{\theta_{x}\left(\tau_{2}, x\right)}{\theta\left(\tau_{2}, x\right)} f\left(\tau_{2}\right)-\frac{\theta_{x}\left(\tau_{1}, x\right)}{\theta\left(\tau_{1}, x\right)} f\left(\tau_{2}\right)-\frac{\theta_{x}\left(\tau_{2}, x\right)}{\theta\left(\tau_{2}, x\right)} f\left(\tau_{1}\right)\right] d \tau_{1} d \tau_{2} \\
& =\int_{0 \leq \tau_{1} \leq \tau_{2} \leq T} \phi\left(\tau_{1}\right) \phi\left(\tau_{2}\right)\left[\frac{\theta_{x}\left(\tau_{2}, x\right)}{\theta\left(\tau_{2}, x\right)}-\frac{\theta_{x}\left(\tau_{1}, x\right)}{\theta\left(\tau_{1}, x\right)}\right]\left[f\left(\tau_{2}\right)-f\left(\tau_{1}\right)\right] d \tau_{1} d \tau_{2}>0
\end{aligned}
$$

where $\phi(\tau) \equiv \alpha(\tau) \tau \theta(\tau, x), f(\tau) \equiv \frac{1-e^{-\nu_{2} \tau}}{\nu_{2}}$, and the last inequality follows because $\theta_{x}(\tau, x) / \theta(\tau, x)$ and $f(\tau)$ are increasing in $\tau$.

Since $\bar{\nu}_{2}$ is decreasing in $x$, it converges to limits when $x$ converges to $\underline{x}$ and $\bar{x}$. These limits must be $\infty$ and $-\infty$, respectively. Indeed, since $\lim _{x \rightarrow \underline{x}} \frac{\int_{\epsilon}^{T} \alpha(\tau) \tau \theta(\tau, x) d \tau}{\int_{0}^{T} \alpha(\tau) \tau \theta(\tau, x) d t}=0$ for all $\epsilon>0$,

$$
\lim _{x \rightarrow \underline{x}} \frac{\int_{0}^{T} \alpha(\tau) \tau \theta(\tau, x) \frac{1-e^{-\nu_{2} \tau}}{\nu_{2}} d \tau}{\int_{0}^{T} \alpha(\tau) \tau \theta(\tau, x) d \tau}=0
$$

and therefore, the left-hand side of (A.52) is negative for $x \rightarrow \underline{x}$ and any finite $\nu_{2}$. Likewise, since $\lim _{x \rightarrow \bar{x}} \frac{\int_{0}^{T-\epsilon} \alpha(\tau) \tau \theta(\tau, x) d \tau}{\int_{0}^{T} \alpha(\tau) \tau \theta(\tau, x) d t}=0$ for all $\epsilon>0$,

$$
\lim _{x \rightarrow \bar{x}} \frac{\int_{0}^{T} \alpha(\tau) \tau \theta(\tau, x) \frac{1-e^{-\nu_{2} \tau}}{\nu_{2}} d \tau}{\int_{0}^{T} \alpha(\tau) \tau \theta(\tau, x) d \tau}=\frac{1-e^{-\nu_{2} T}}{\nu_{2}},
$$

and therefore, the left-hand side of (A.52) is positive for $x \rightarrow \bar{x}$ and any finite $\nu_{2}$. Therefore, there exists $x^{*} \in(\underline{x}, \bar{x})$ such that $\bar{\nu}_{2}>0$ if $x<x^{*}$ and $\bar{\nu}_{2}<0$ if $x>x^{*}$.

Propositions 4 and 5 imply that when $a \approx \infty, A_{\beta}^{\prime}(\tau)$ is increasing and then decreasing if $\nu_{2}>0$, and is increasing if $\nu_{2}<0$. The maximum of $A_{\beta}^{\prime}(\tau)$ in the first case is

$$
\frac{\log \nu_{1}-\log \nu_{2}}{\nu_{1}-\nu_{2}} \approx \frac{\log \left(a^{\frac{1}{3}} \bar{n}_{1}\right)-\log \bar{\nu}_{2}}{a^{\frac{1}{3}} \bar{n}_{1}-\bar{\nu}_{2}} \approx \frac{\log \left(a^{\frac{1}{3}}\right)}{a^{\frac{1}{3}} \bar{n}_{1}}
$$

and is in $(0, T)$. Therefore, $A_{\beta}^{\prime}(\tau)$ is increasing and then decreasing in $[0, T]$ if $x<x^{*}$, and is increasing in $[0, T]$ if $x>x^{*}$. 
Proof of Corollary 3: The shock raises instantaneous forward rates because $A_{\beta}^{\prime}(\tau)>0$ for all $\tau$. It also raises spot rates because $A_{\beta}(\tau)=\int_{0}^{T} A_{\beta}^{\prime}(\tau) d \tau>0$ for all $\tau$. The rest of the corollary follows from (36), (38) and Proposition 6.

Proof of Proposition 7: Eqs. (A.14) and (A.15) imply that $\left(\lambda_{r, t}, \lambda_{\beta, t}\right)$ are affine in $\left(r_{t}, \beta_{t}\right)$. Combining (A.14) and (A.15) with (31), we find

$$
\begin{aligned}
& \frac{\partial \lambda_{r, t}}{\partial r_{t}}=-a \sigma_{r}^{2} \int_{0}^{T} \alpha(\tau) A_{r}(\tau)^{2} d \tau=M_{1,1}<0, \\
& \frac{\partial \lambda_{r, t}}{\partial \beta_{t}}=a \sigma_{r}^{2} \int_{0}^{T} \alpha(\tau)\left[\tau \theta(\tau)-A_{\beta}(\tau)\right] A_{r}(\tau) d \tau=M_{2,1}, \\
& \frac{\partial \lambda_{\beta, t}}{\partial r_{t}}=-a \sigma_{\beta}^{2} \int_{0}^{T} \alpha(\tau) A_{r}(\tau) A_{\beta}(\tau) d \tau=M_{1,2}, \\
& \frac{\partial \lambda_{\beta, t}}{\partial \beta_{t}}=a \sigma_{\beta}^{2} \int_{0}^{T} \alpha(\tau)\left[\tau \theta(\tau)-A_{\beta}(\tau)\right] A_{\beta}(\tau) d \tau=M_{2,2} .
\end{aligned}
$$

When $a \approx 0$, Propositions 4 and 5 imply that

$$
\begin{aligned}
A_{r}(\tau) & =\frac{1-e^{-\kappa_{r} \tau}}{\kappa_{r}}+o(1), \\
A_{\beta}(\tau) & =a \underline{c}_{\beta}\left(\frac{1-e^{-\kappa_{\beta}}}{\kappa_{\beta}}-\frac{1-e^{-\kappa_{r}}}{\kappa_{r}}\right)+o(a) \\
& =\frac{a \sigma_{r}^{2} \int_{0}^{T} \alpha(\tau) \tau \theta(\tau) \frac{1-e^{-\kappa_{r} \tau}}{\kappa_{r}} d \tau}{\kappa_{r}-\kappa_{\beta}}\left(\frac{1-e^{-\kappa_{\beta}}}{\kappa_{\beta}}-\frac{1-e^{-\kappa_{r}}}{\kappa_{r}}\right)+o(a),
\end{aligned}
$$

where the second step in (A.58) follows from (A.38). Substituting (A.57) and (A.58) into (A.54)(A.56), and using $\theta(\tau)>0$ for all $\tau$, we find $M_{1,2}<0, M_{2,1}>0$ and $M_{2,2}>0$. When $a \approx \infty$, Proposition 5 and (A.24)-(A.26) imply that

$$
\begin{aligned}
& M_{1,2}=\frac{\bar{c}_{r} \bar{n}_{1}}{\bar{\gamma}_{\beta}}+o(1), \\
& M_{2,1}=a^{\frac{1}{3}} \bar{\gamma}_{\beta} \bar{n}_{1}+o\left(a^{\frac{1}{3}}\right), \\
& M_{2,2}=\kappa_{\beta}-\bar{\nu}_{2}-\bar{c}_{r} \bar{n}_{1}+o(1)=\kappa_{\beta}+\bar{\nu}_{2}\left[\frac{\int_{0}^{T} \alpha(\tau)\left(1-e^{-\bar{\nu}_{2} \tau}\right) d \tau}{\int_{0}^{T} \alpha(\tau)\left(1-e^{-\bar{\nu}_{2} \tau}\right)^{2} d \tau}-1\right]+o(1),
\end{aligned}
$$

where the second step in (A.61) follows from (A.50). Since $\theta(\tau)>0$ for all $\tau$, (A.51) implies that $\bar{\gamma}_{\beta}>0$. Since, in addition, $\bar{n}_{1}>0$ and $\bar{c}_{r}<0$, (A.59) and (A.60) imply that $M_{1,2}<0$ and $M_{2,1}>0$. Eq. $M_{2,2}>0$ follows from (A.61) because the term in square brackets has the same sign as $\bar{\nu}_{2}$. 
Since the risk premium of the bond with maturity $\tau$ is $A_{r}(\tau) \lambda_{r, t}+A_{\beta}(\tau) \lambda_{\beta, t}$, it is affine in $\left(r_{t}, \beta_{t}\right)$. When $a \approx 0$, the risk premium is decreasing in $r_{t}$ and increasing in $\beta_{t}$ because the same comparative statics hold for $\left(\lambda_{r, t}, \lambda_{\beta, t}\right)$ and because (A.57) and (A.58) imply that $A_{r}(\tau), A_{\beta}(\tau)>0$. To show the comparative statics for $a \approx \infty$, we recall from (26) that the risk premium is $\mu_{t, \tau}-r_{t}$. Using (25) and Propositions 4 and 5, we find

$$
\begin{aligned}
& \frac{\partial\left(\mu_{t, \tau}-r_{t}\right)}{\partial r_{t}}=A_{r}^{\prime}(\tau)+\kappa_{r} A_{r}(\tau)-1=-1+o(1)<0 \\
& \frac{\partial\left(\mu_{t, \tau}-r_{t}\right)}{\partial \beta_{t}}=A_{\beta}^{\prime}(\tau)+\kappa_{\beta} A_{\beta}(\tau)=\bar{\gamma}_{\beta}\left(e^{-\bar{\nu}_{2} \tau}+\kappa_{\beta} \frac{1-e^{-\bar{\nu}_{2} \tau}}{\bar{\nu}_{2}}\right)+o(1)>0 .
\end{aligned}
$$

Therefore, the risk premium is decreasing in $r_{t}$ and increasing in $\beta_{t}$.

Proof of Proposition 8: Proceeding as in the proof of Proposition 2, we find that in the limit when $\Delta \tau \rightarrow 0$,

$$
\begin{aligned}
\gamma_{\mathrm{FB}} & \rightarrow \frac{\left[A_{r}^{\prime}(\tau)+\kappa_{r} A_{r}(\tau)-1\right]\left[A_{r}^{\prime}(\tau)-1\right] \operatorname{Var}\left(r_{t}\right)+\left[A_{\beta}^{\prime}(\tau)+\kappa_{\beta} A_{\beta}(\tau)\right] A_{\beta}^{\prime}(\tau) \operatorname{Var}\left(\beta_{t}\right)}{\left[A_{r}^{\prime}(\tau)-1\right]^{2} \operatorname{Var}\left(r_{t}\right)+A_{\beta}^{\prime}(\tau)^{2} \operatorname{Var}\left(\beta_{t}\right)} \\
= & \frac{\left[A_{r}^{\prime}(\tau)+\kappa_{r} A_{r}(\tau)-1\right]\left[A_{r}^{\prime}(\tau)-1\right] \frac{\sigma_{r}^{2}}{\kappa_{r}}+\left[A_{\beta}^{\prime}(\tau)+\kappa_{\beta} A_{\beta}(\tau)\right] A_{\beta}^{\prime}(\tau) \frac{\sigma_{\beta}^{2}}{\kappa_{\beta}}}{\left[A_{r}^{\prime}(\tau)-1\right]^{2} \frac{\sigma_{r}^{2}}{\kappa_{r}}+A_{\beta}^{\prime}(\tau)^{2} \frac{\sigma_{\beta}^{2}}{\kappa_{\beta}}} .
\end{aligned}
$$

When $a \approx 0$, Propositions 4 and 5 imply that the right-hand side of (A.64) is

$$
\frac{\nu_{1}-\kappa_{r}}{\nu_{1}}+\frac{a^{2} \underline{c}_{\beta}^{2}\left(\kappa_{r}-\kappa_{\beta}\right)\left(e^{-\kappa_{\beta} \tau}-e^{-\kappa_{r} \tau}\right) \frac{\sigma_{\beta}^{2}}{\kappa_{\beta}}}{\left(1-e^{-\kappa_{r} \tau}\right) \frac{\sigma_{r}^{2}}{\kappa_{r}}}+o\left(a^{2}\right)
$$

Proposition 5 and (A.27) imply that $\nu_{1}-\kappa_{r}$ is of order $a$, positive, and independent of $\sigma_{\beta}^{2}$ up to order $a^{3}$. Therefore, $\gamma_{\mathrm{FB}}$ is positive and increasing in $\sigma_{\beta}^{2}$.

When $a \approx \infty,($ A.62) and (A.63) imply that the right-hand side of (A.64) is

$$
\frac{\frac{\sigma_{r}^{2}}{\kappa_{r}}+\bar{\gamma}_{\beta}^{2}\left(e^{-\bar{\nu}_{2} \tau}+\kappa_{\beta} \frac{1-e^{-\bar{\nu}_{2} \tau}}{\bar{\nu}_{2}}\right) e^{-\bar{\nu}_{2} \tau} \frac{\sigma_{\beta}^{2}}{\kappa_{\beta}}}{\frac{\sigma_{r}^{2}}{\kappa_{r}}+\bar{\gamma}_{\beta}^{2} e^{-2 \bar{\nu}_{2} \tau} \frac{\sigma_{\beta}^{2}}{\kappa_{\beta}}}+o(1) .
$$

Therefore, $\gamma_{\mathrm{FB}}$ is positive and increasing in $\sigma_{\beta}^{2}$. 
Proof of Proposition 9: Proceeding as in the proof of Propositions 2 and 8, we find

$$
\begin{aligned}
\gamma_{\mathrm{CS}} & \rightarrow \frac{\left[\frac{A_{r}(\tau)}{\tau}-\left[A_{r}^{\prime}(\tau)+\kappa_{r} A_{r}(\tau)\right]\right]\left[\frac{A_{r}(\tau)}{\tau}-1\right] \operatorname{Var}\left(r_{t}\right)+\left[\frac{A_{\beta}(\tau)}{\tau}-\left[A_{\beta}^{\prime}(\tau)+\kappa_{\beta} A_{\beta}(\tau)\right]\right] \frac{A_{\beta}(\tau)}{\tau} \operatorname{Var}\left(\beta_{t}\right)}{\left[\frac{A_{r}(\tau)}{\tau}-1\right]^{2} \operatorname{Var}\left(r_{t}\right)+\left[\frac{A_{\beta}(\tau)}{\tau}\right]^{2} \operatorname{Var}\left(\beta_{t}\right)} \\
& =1-\frac{\left[A_{r}^{\prime}(\tau)+\kappa_{r} A_{r}(\tau)-1\right]\left[\frac{A_{r}(\tau)}{\tau}-1\right] \frac{\sigma_{r}^{2}}{\kappa_{r}}+\left[A_{\beta}^{\prime}(\tau)+\kappa_{\beta} A_{\beta}(\tau)\right] \frac{A_{\beta}(\tau)}{\tau} \frac{\sigma_{\beta}^{2}}{\kappa_{\beta}}}{\left[\frac{A_{r}(\tau)}{\tau}-1\right]^{2} \frac{\sigma_{r}^{2}}{\kappa_{r}}+\left[\frac{A_{\beta}(\tau)}{\tau}\right]^{2} \frac{\sigma_{\beta}^{2}}{\kappa_{\beta}}}
\end{aligned}
$$

When $a \approx 0$, Propositions 4 and 5 imply that the right-hand side of (A.65) is

$$
1-\frac{\left(\nu_{1}-\kappa_{r}\right)\left(1-e^{-\kappa_{r} \tau}\right)}{\kappa_{r}\left(1-\frac{1-e^{-\kappa_{r} \tau}}{\kappa_{r} \tau}\right)}+o(a)
$$

Since $\nu_{1}-\kappa_{r}$ is of order $a$ and positive, $\gamma_{\mathrm{CS}}$ is smaller than and close to one. Moreover, $\gamma_{\mathrm{CS}}$ is increasing in $\tau$ if the function

$$
f(x) \equiv \frac{1-\frac{1-e^{-x}}{x}}{1-e^{-x}}
$$

is increasing in $x \geq 0$. The derivative $f^{\prime}(x)$ has the same sign as the function

$$
g(x) \equiv 1-e^{-x}-x e^{-\frac{x}{2}},
$$

and the derivative $g^{\prime}(x)$ has the same sign as

$$
e^{-\frac{x}{2}}-1+\frac{x}{2}>0
$$

Therefore, $g(x)>0$ and $f(x)$ is increasing in $x$.

When $a \approx \infty,($ A.62) and (A.63) imply that the right-hand side of (A.65) is

$$
1-\frac{\frac{\sigma_{r}^{2}}{\kappa_{r}}+\bar{\gamma}_{\beta}^{2}\left(e^{-\bar{\nu}_{2} \tau}+\kappa_{\beta} \frac{1-e^{-\bar{\nu}_{2} \tau}}{\bar{\nu}_{2}}\right) \frac{1-e^{-\bar{\nu}_{2} \tau}}{\bar{\nu}_{2} \tau} \frac{\sigma_{\beta}^{2}}{\kappa_{\beta}}}{\frac{\sigma_{r}^{2}}{\kappa_{r}}+\bar{\gamma}_{\beta}^{2}\left(\frac{1-e^{-\bar{\nu}_{2} \tau}}{\bar{\nu}_{2} \tau}\right)^{2} \frac{\sigma_{\beta}^{2}}{\kappa_{\beta}}}+o(1) .
$$

Therefore, $\gamma_{\mathrm{CS}}$ is smaller than one. To show that $\gamma_{\mathrm{CS}}$ can be negative and decreasing in $\tau$, suppose that $\bar{\nu}_{2}<0$ (which Proposition 6 shows to happen when $\theta(\tau)$ gives high weight to long maturities). Since the second term in (A.66) converges to $\infty$ when $\tau \rightarrow \infty$, (A.65) and (A.66) imply that $\gamma_{\mathrm{CS}}$ is negative and decreasing in $\tau$ for large $\tau$. 
Proof of Lemma 3: Applying Ito's Lemma to (42) and using the dynamics (3) of $r_{t}$ and (6) of $\beta_{k, t}$, we find

$$
\frac{d P_{t, \tau}}{P_{t, \tau}}=\mu_{t, \tau} d t-A_{r}(\tau) \sigma_{r} d B_{r, t}-\sum_{k=1}^{K} A_{\beta, k}(\tau) \sigma_{\beta, k} d B_{\beta, k, t}
$$

where

$$
\begin{aligned}
\mu_{t, \tau} \equiv & A_{r}^{\prime}(\tau) r_{t}+\sum_{k=1}^{K} A_{\beta, k}^{\prime}(\tau) \beta_{k, t}+C^{\prime}(\tau)-A_{r}(\tau) \kappa_{r}\left(\bar{r}-r_{t}\right)+\sum_{k=1}^{K} A_{\beta, k}(\tau) \kappa_{\beta, k} \beta_{k, t} \\
& +\frac{1}{2} A_{r}(\tau)^{2} \sigma_{r}^{2}+\frac{1}{2} \sum_{k=1}^{K} A_{\beta, k}(\tau)^{2} \sigma_{\beta, k}^{2} .
\end{aligned}
$$

Using (A.67) and proceeding as in Lemmas 1 and 2, we can write (8) as

$$
\max _{\left\{x_{t, \tau}\right\}_{\tau \in(0, T]}}\left[\int_{0}^{T} x_{t, \tau}\left(\mu_{t, \tau}-r_{t}\right) d \tau-\frac{a \sigma_{r}^{2}}{2}\left[\int_{0}^{T} x_{t, \tau} A_{r}(\tau) d \tau\right]^{2}-\frac{a}{2} \sum_{k=1}^{K} \sigma_{\beta, k}^{2}\left[\int_{0}^{T} x_{t, \tau} A_{\beta, k}(\tau) d \tau\right]^{2}\right] .
$$

Point-wise maximization of (A.69) yields (43).

Proof of Proposition 10: Proceeding as in Propositions 1 and 4, we find that the functions $A_{r}(\tau),\left\{A_{\beta, k}(\tau)\right\}_{k=1, . ., K}$ solve the system of

$$
\begin{aligned}
& A_{r}^{\prime}(\tau)+\kappa_{r} A_{r}(\tau)-1=A_{r}(\tau) M_{1,1}+\sum_{l=1}^{K} A_{\beta, l}(\tau) M_{1, l+1}, \\
& A_{\beta, k}^{\prime}(\tau)+\kappa_{\beta, k} A_{\beta, k}(\tau)=A_{r}(\tau) M_{k+1,1}+\sum_{l=1}^{K} A_{\beta, l}(\tau) M_{k+1, l+1},
\end{aligned}
$$

where

$$
\begin{aligned}
M_{1,1} & \equiv-\sigma_{r}^{2} \int_{0}^{T} \alpha(\tau) A_{r}(\tau)^{2} d \tau \\
M_{1, l+1} & \equiv-\sigma_{\beta}^{2} \int_{0}^{T} \alpha(\tau) A_{r}(\tau) A_{\beta, l}(\tau) d \tau \\
M_{k+1,1} & \equiv \sigma_{r}^{2} \int_{0}^{T} \alpha(\tau)\left[\tau \theta_{k}(\tau)-A_{\beta, k}(\tau)\right] A_{r}(\tau) d \tau \\
M_{k+1, l+1} & \equiv \sigma_{\beta}^{2} \int_{0}^{T} \alpha(\tau)\left[\tau \theta_{k}(\tau)-A_{\beta, k}(\tau)\right] A_{\beta, l}(\tau) d \tau,
\end{aligned}
$$


for $k, l=1, . ., K$, and the function $C(\tau)$ solves

$$
\begin{aligned}
& C^{\prime}(\tau)-\kappa_{r} \bar{r} A_{r}(\tau)+\frac{1}{2} \sigma_{r}^{2} A_{r}(\tau)^{2}+\frac{1}{2} \sum_{k=1}^{K} \sigma_{\beta, k}^{2} A_{\beta, k}(\tau)^{2} \\
& =a \sigma_{r}^{2} A_{r}(\tau) \int_{0}^{T} \alpha(\tau)[\bar{\beta} \tau-C(\tau)] A_{r}(\tau) d \tau+a \sum_{k=1}^{K} \sigma_{\beta, k}^{2} A_{\beta, k}(\tau) \int_{0}^{T} \alpha(\tau)[\bar{\beta} \tau-C(\tau)] A_{\beta, k}(\tau) d \tau .
\end{aligned}
$$

The differential equations (A.70), (A.71) and (A.76) must be solved with the initial conditions $A_{r}(0)=A_{\beta, k}(0)=C(0)=0$. The same argument as in the proof of Proposition 4 implies that the solution to the system of (A.70)-(A.71) has the form in the proposition. To determine the scalars $\left\{\nu_{l}\right\}_{l=1, . ., K+1},\left\{\gamma_{r, l}\right\}_{l=1, . ., K},\left\{\gamma_{\beta, k, l}\right\}_{k, l=1, . ., K}$, we substitute (46) and (47) into (A.70) and (A.71), and identify terms in $\frac{1-e^{-\nu_{l} \tau}}{\nu_{l}}$ for $l=1, . ., K+1$. This yields

$$
\begin{aligned}
& \left(1-\sum_{l=1}^{K} \gamma_{r, l}\right)\left(\nu_{1}-\kappa_{r}+M_{1,1}\right)-\sum_{l, l^{\prime}=1}^{K} \gamma_{\beta, l^{\prime}, l} M_{1, l^{\prime}+1}=0, \\
& \gamma_{r, l}\left(\nu_{l+1}-\kappa_{r}+M_{1,1}\right)+\sum_{l^{\prime}=1}^{K} \gamma_{\beta, l^{\prime}, l} M_{1, l^{\prime}+1}=0
\end{aligned}
$$

in the case of (A.70) and

$$
\begin{aligned}
& \left(\sum_{l=1}^{K} \gamma_{\beta, k, l}\right)\left(\nu_{1}-\kappa_{\beta}\right)-\left(1-\sum_{l=1}^{K} \gamma_{r, l}\right) M_{k+1,1}+\sum_{l, l^{\prime}=1}^{K} \gamma_{\beta, l^{\prime}, l} M_{k+1, l^{\prime}+1}=0 \\
& -\gamma_{\beta, k, l}\left(\nu_{l+1}-\kappa_{\beta}\right)-\gamma_{r, l} M_{k+1,1}-\sum_{l^{\prime}=1}^{K} \gamma_{\beta, l^{\prime}, l} M_{k+1, l^{\prime}+1}=0
\end{aligned}
$$

in the case of (A.71). Eqs. (A.77)-(A.80) constitute a system of $(K+1)^{2}$ scalar non-linear equations in the unknowns $\left\{\nu_{l}\right\}_{l=1, . ., K+1},\left\{\gamma_{r, l}\right\}_{l=1, . ., K},\left\{\gamma_{\beta, k, l}\right\}_{k, l=1, . ., K}$.

The solution to (A.76) is

$$
C(\tau)=z_{r} \int_{0}^{\tau} A_{r}(u) d u+\sum_{k=1}^{K} z_{\beta, k} \int_{0}^{\tau} A_{\beta, k}(u) d u-\frac{\sigma_{r}^{2}}{2} \int_{0}^{\tau} A_{r}(u)^{2} d u-\sum_{k=1}^{K} \frac{\sigma_{\beta, k}^{2}}{2} \int_{0}^{\tau} A_{\beta, k}(u)^{2} d u
$$


where

$$
\begin{aligned}
z_{r} & \equiv \kappa_{r} \bar{r}+a \sigma_{r}^{2} \int_{0}^{T} \alpha(\tau)[\bar{\beta} \tau-C(\tau)] A_{r}(\tau) d \tau \\
z_{\beta, k} & \equiv a \sigma_{\beta, k}^{2} \int_{0}^{T} \alpha(\tau)[\bar{\beta} \tau-C(\tau)] A_{\beta, k}(\tau) d \tau .
\end{aligned}
$$

Substituting $C(\tau)$ from (A.81) into (A.82) and (A.83), we can derive $z_{r},\left\{z_{\beta, k}\right\}_{k=1, . ., K}$ as the solution to a linear system of equations.

\section{B Demand of Preferred-Habitat Investors}

There are overlapping generations of investors living for a time interval of length $T$, and of arbitrageurs living for a time interval of length $d t$. Thus, at each point in time there is a continuum of investor generations and one arbitrageur generation. There are two goods: good 1 that arbitrageurs and investors can consume and exchange for bonds, and good 2 that investors can produce from good 1, can consume and cannot exchange. Bonds pay in terms of good 1 when they mature.

Arbitrageurs and investors receive endowment $W$ of good 1 at the beginning of their life. Arbitrageurs use their endowment to buy bonds, and at the end of their life (i.e., after $d t$ ), they sell the bonds and consume good 1. Investors use their endowment to buy bonds, and can also invest good 1 in a private technology at any time prior to the end of their life. The technology yields good 2 at the end of investors' life, and investors consume goods 1 and 2 at that time.

For notational simplicity, we consider an investor born at time 0 . At time $t<T$, the investor holds $\hat{y}_{t, \tau}$ units of the bond with maturity $\tau$ and invests $d c_{t}$ units of good 1 in the private technology.

The investment in the private technology yields $e^{\hat{\beta}_{t}(T-t)} d c_{t}$ units of good 2 at time $T$. The return $\hat{\beta}_{t}$ is

$$
\hat{\beta}_{t}=\beta_{t, T-t}+\eta_{t},
$$

where $\beta_{t, T-t}$ is given by (5) and $\eta_{t}$ is investor-specific noise that averages out over the investor's generational cohort. The noise follows the Ornstein-Uhlenbeck process

$$
d \eta_{t}=-\kappa_{\eta} \eta_{t}+\sigma_{\eta} d B_{\eta, t}
$$

where $\left(\kappa_{\eta}, \sigma_{\eta}\right)$ are positive constants and $B_{\eta, t}$ is a Brownian motion independent of all other uncertainty in the model. 
We write the investor's budget constraint using as numeraire the bond maturing at time $T$. Denoting by $\hat{W}_{t}$ the investor's wealth expressed in units of that bond, the budget constraint is

$$
d \hat{W}_{t}=\int_{0}^{T} \hat{y}_{t, \tau} d\left(\frac{P_{t, \tau}}{P_{t, T-t}}\right) d \tau-\frac{d c_{t}}{P_{t, T-t}} .
$$

The investor's utility at time $T$ is

$$
u\left(C_{T}\right)+\int_{0}^{T} e^{\hat{\beta}_{t}(T-t)} d c_{t}
$$

where the first and second terms represent utility derived from consumption of good 1 and 2 , respectively. The utility $u\left(C_{T}\right)$ derived from consumption of good 1 is an increasing and concave function of $C_{T}$. The utility derived from consumption of good 2 is equal to the consumption of that good, which is derived from the accumulated investment in the private technology. ${ }^{28}$ The investor has max-min preferences and at time 0 solves the optimization problem

$$
\max _{\hat{y}_{t, \tau}, c_{t}} \min _{r_{t}, \beta_{t}, \eta_{t}}\left[u\left(C_{T}\right)+\int_{0}^{T} e^{\hat{\beta}_{t}(T-t)} d c_{t}\right]
$$

subject to the budget constraint (B.1) and the terminal condition $C_{T}=\hat{W}_{T}$. The minimum in (B.3) is over sample paths of $\left(r_{t}, \beta_{t}, \eta_{t}\right)$, where $\beta_{t} \equiv\left(\beta_{1, t}, . ., \beta_{K, t}\right)$, and the maximum is over adapted controls $\left(\hat{y}_{t, \tau}, c_{t}\right)$.

Proposition B.1. Assume that the term structure involves no arbitrage, i.e., (43) holds. At time $t$, the investor holds

$$
\hat{y}_{t, T-t}=\left(u^{\prime}\right)^{-1}\left[P_{t, T-t} e^{\hat{\beta}_{t}(T-t)}\right]
$$

units of the bond maturing at time $T$ and zero units of all other bonds, or a portfolio replicating this position.

Proof: We can write the budget constraint (B.1) as

$$
d \hat{W}_{t}=\mu_{\hat{y}, t} d t+\sigma_{\hat{y}, t} d B_{t}-d \hat{c}_{t},
$$

\footnotetext{
${ }^{28} \mathrm{An}$ alternative interpretation of (B.2) is that there is no good 2 and the second term represents utility derived from intermediate consumption of good 1. Under this interpretation, $e^{\hat{\beta}_{t}(T-t)}$ is a stochastic preference factor for consumption of good 1 at time $t$ relative to $T$.
} 
where

$$
\begin{aligned}
& \int_{0}^{T} \hat{y}_{t, \tau} d\left(\frac{P_{t, \tau}}{P_{t, T-t}}\right) d \tau \equiv \mu_{\hat{y}, t} d t+\sigma_{\hat{y}, t} d B_{t}, \\
& B_{t}^{\prime} \equiv\left(B_{r, t}, B_{\beta, 1, t}, . ., B_{\beta, K, t}\right), \\
& \hat{c}_{t} \equiv \frac{d c_{t}}{P_{t, T-t}} .
\end{aligned}
$$

Integrating (B.5) from 0 to $T$ and using the terminal condition $C_{T}=\hat{W}_{T}$, we can write the investor's optimization problem (B.3) as

$$
\max _{\hat{y} t, \tau, c_{s}} \min _{r_{t}, \beta_{t}, \eta_{t}}\left[u\left(\hat{W}_{0}+\int_{0}^{T} \mu_{\hat{y}, t} d t+\int_{0}^{T} \sigma_{\hat{y}, t} d B_{t}-\int_{0}^{T} d \hat{c}_{t}\right)+\int_{0}^{T} z_{t} d \hat{c}_{t}\right]
$$

where

$$
z_{t} \equiv P_{t, T-t} e^{\hat{\beta}_{t}(T-t)}
$$

For any sample path of $\left(r_{t}, \beta_{t}\right)$ and positive constant $g$, the sample path where $z_{t}=g$ for $t>0$ is possible because $\eta_{t}$ is independent of all other uncertainty in the model and can take any value. ${ }^{29}$ Therefore,

$$
\begin{aligned}
& \min _{r_{t}, \beta_{t}, \eta_{t}}\left[u\left(\hat{W}_{0}+\int_{0}^{T} \mu_{\hat{y}, t} d t+\int_{0}^{T} \sigma_{\hat{y}, t} d B_{t}-\int_{0}^{T} d \hat{c}_{t}\right)+\int_{0}^{T} z_{t} d \hat{c}_{t}\right] \\
& \leq \min _{r_{t}, \beta_{t}}\left[u\left(\hat{W}_{0}+\int_{0}^{T} \mu_{\hat{y}, t} d t+\int_{0}^{T} \sigma_{\hat{y}, t} d B_{t}-d \hat{c}_{0}-\int_{0^{+}}^{T} d \hat{c}_{t}\right)+z_{0} d \hat{c}_{0}+\int_{0^{+}}^{T} u^{\prime}\left(\hat{W}_{0}-d \hat{c}_{0}\right) d \hat{c}_{t}\right] \\
& \leq \min _{r_{t}, \beta_{t}}\left[u\left(\hat{W}_{0}-d \hat{c}_{0}\right)+u^{\prime}\left(\hat{W}_{0}-d \hat{c}_{0}\right)\left(\int_{0}^{T} \mu_{\hat{y}, t} d t+\int_{0}^{T} \sigma_{\hat{y}, t} d B_{t}\right)+z_{0} d \hat{c}_{0}\right],
\end{aligned}
$$

where $d \hat{c}_{0}$ denotes a possible atom of $d \hat{c}_{t}$ at zero, $\int_{0^{+}}^{T}$ denotes integrals excluding the atom, the first step follows by setting $g=u^{\prime}\left(\hat{W}_{0}-d \hat{c}_{t}\right)$, and the second step follows from the concavity of $u$. If $\sigma_{\hat{y}, t} \neq 0$, then the minimum in (B.7) is $-\infty$ because the Brownian motion has infinite variation. Therefore, $\sigma_{\hat{y}, t}=0$, i.e., the investor holds the bond maturing at time $T$ and zero units of all other bonds, or a portfolio replicating this position. Since absence of arbitrage requires that $\mu_{\hat{y}, t}=0$,

\footnotetext{
${ }^{29}$ More precisely, the sample path where $z_{t}=g$ for $t>\epsilon$ is possible for $\epsilon$ arbitrarily close to 0 .
} 
(B.7) implies that

$$
\begin{aligned}
& \max _{\hat{y}_{t, \tau}, c_{t}} \min _{r_{t}, \beta_{t}, \eta_{t}}\left[u\left(\hat{W}_{0}+\int_{0}^{T} \mu_{\hat{y}, t} d t+\int_{0}^{T} \sigma_{\hat{y}, t} d B_{t}-\int_{0}^{T} d \hat{c}_{t}\right)+\int_{0}^{T} z_{t} d \hat{c}_{t}\right] \\
& \leq \max _{d \hat{c}_{0}}\left[u\left(\hat{W}_{0}-d \hat{c}_{0}\right)+z_{0} d \hat{c}_{0}\right] .
\end{aligned}
$$

Setting $y_{t, \tau}=0$ for $t \geq 0$ and $\tau \neq T-t$, and $d \hat{c}_{t}=0$ for $t>0$, in (B.6), we find that (B.8) holds also in the reverse sense, and is therefore an equality. The optimal $d \hat{c}_{0}$ thus satisfies

$$
u^{\prime}\left(\hat{W}_{0}-d \hat{c}_{0}\right)=z_{0}
$$

Since $\hat{W}_{0}-d \hat{c}_{0}$ represents units of the bond maturing at time $T$ that the investor holds at time 0 , (B.9) yields (B.4) for $t=0$. The same argument yields (B.4) for $t>0$.

To derive the demand (4), we set $u\left(C_{T}\right)=-e^{-C_{T}}$. Proposition (B.1) implies that the investor holds

$$
-\log \left(e^{\hat{\beta}_{t}(T-t)} P_{t, T-t}\right)=(T-t)\left(R_{t, T-t}-\hat{\beta}_{t}\right)
$$

units of the bond that matures at time $T$. Therefore, the average demand across the generational cohort is for

$$
y_{t, T-t}=(T-t)\left(R_{t, T-t}-\beta_{t, T-t}\right)
$$

units of the bond, and has the form (4) with $\alpha(\tau)=1$.

We next present a modification of our model where the demand of preferred-habitat investors is derived from optimizing behavior and used - in its exact form - in the determination of equilibrium bond prices. The modified model makes use of Gabaix's (2009) linearity-generating processes. For expositional simplicity, we consider the case where the short rate is the only stochastic factor $(K=0)$. We assume that the short rate follows the linearity-generating process

$$
d r_{t}=\left(\kappa_{r}+\bar{r}-r_{t}\right)\left(\bar{r}-r_{t}\right) d t+d N_{r, t}
$$

where $\left(\bar{r}, \kappa_{r}\right)$ are positive constants and $N_{r, t}$ is a martingale. To use the results of Cheridito and Gabaix (2008) and Gabaix (2009), we must ensure that $r_{t}$ remains below a bound $\hat{r} \in\left(\bar{r}, \bar{r}+\kappa_{r}\right)$. Moreover, our analysis requires that the instantaneous variance of the short rate is equal to a 
constant $\sigma_{r}^{2}$. The two requirements cannot be met simultaneously if $N_{r, t}$ is a Brownian motion, but can be met by introducing a jump component alongside the Brownian component. ${ }^{30}$

We set $u\left(C_{T}\right)=g C_{T}-\frac{1}{2} C_{T}^{2}$ for $C_{T} \leq g$ and $u\left(C_{T}\right)=g^{2} / 2$ for $C_{T}>g$, where $g$ is a positive constant. Proposition B.1 implies that at time $t$ the investor holds

$$
g-e^{\left(\bar{\beta}+\eta_{t}\right)(T-t)} P_{t, T-t}
$$

units of the bond that matures at time $T .{ }^{31}$ Therefore, the average demand across the generational cohort is for

$$
y_{t, T-t}=g-f(T-t) P_{t, T-t}
$$

units of the bond, where $f(\tau) \equiv e^{\bar{\beta} \tau+\frac{1}{2} \Sigma_{\eta}^{2} \tau^{2}}$ and $\Sigma_{\eta}^{2}$ is the intra-generational variance of $\eta_{t}$. We take $\Sigma_{\eta}^{2}$ to be constant over time and equal to its steady-state value.

We conjecture that equilibrium bond prices are affine in $r_{t}$, i.e.,

$$
P_{t, \tau}=C(\tau)-A_{r}(\tau) r_{t}
$$

for two functions $A_{r}(\tau), C(\tau)$ that depend on maturity $\tau$. Eqs. (B.10) and (B.13) yield the following counterpart of (10):

$$
d P_{t, \tau}=\mu_{t, \tau} d t-A_{r}(\tau) d N_{r, t},
$$

where

$$
\mu_{t, \tau} \equiv-\left[C^{\prime}(\tau)-A_{r}^{\prime}(\tau) r_{t}\right]-A_{r}(\tau)\left(\kappa_{r}+\bar{r}-r_{t}\right)\left(\bar{r}-r_{t}\right)
$$

We write the arbitrageurs' budget constraint in terms of bond units rather than dollar investment. Denoting by $x_{t, \tau}$ the units of bond with maturity $\tau$ held by arbitrageurs at time $t$, the budget constraint is

$$
d W_{t}=\left(W_{t}-\int_{0}^{T} x_{t, \tau} P_{t, \tau}\right) r_{t} d t+\int_{0}^{T} x_{t, \tau} d P_{t, \tau} .
$$

\footnotetext{
${ }^{30}$ We are grateful to Xavier Gabaix for providing to us the process $N_{r, t}$. The construction of $N_{r, t}$ is available upon request.

${ }^{31}$ Proposition B.1 can be generalized to accommodate linearity-generating processes and jump components. Eq (B.11) assumes that $P_{t, T-t}>0$, which is the case in equilibrium because of no-arbitrage and bonds' positive payoff.
} 
The arbitrageurs' first-order condition is

$$
\mu_{t, \tau}-r_{t} P_{t, \tau}=A_{r}(\tau) \lambda_{r, t}
$$

where $\lambda_{r, t}$ is given by (13). Setting $x_{t, \tau}=-y_{t, \tau}$ in (13), we can write $\lambda_{r, t}$ as

$$
\lambda_{r, t}=-a \sigma_{r}^{2} \int_{0}^{T} y_{t, \tau} A_{r}(\tau) d \tau=a \sigma_{r}^{2} \int_{0}^{T}\left[f(\tau)\left[C(\tau)-A_{r}(\tau) r_{t}\right]-g\right] A_{r}(\tau) d \tau,
$$

where the second step follows from (B.12) and (B.13). Substituting $\mu_{t, \tau}$ from (B.15) and $\lambda_{r, t}$ from (B.17) into (B.16), we find

$$
\begin{aligned}
& -\left[C^{\prime}(\tau)-A_{r}^{\prime}(\tau) r_{t}\right]-A_{r}(\tau)\left(\kappa_{r}+\bar{r}-r_{t}\right)\left(\bar{r}-r_{t}\right)-r_{t}\left[C(\tau)-A_{r}(\tau) r_{t}\right] \\
& =A_{r}(\tau) a \sigma_{r}^{2} \int_{0}^{T}\left[f(\tau)\left[C(\tau)-A_{r}(\tau) r_{t}\right]-g\right] A_{r}(\tau) d \tau .
\end{aligned}
$$

Eq. (B.18) is affine in $r_{t}$. Setting linear terms in $r_{t}$ and constant terms to zero yields two linear differential equations in $A_{r}(\tau), C(\tau)$. 


\section{References}

Bakshi, G. and Z. Chen, 1996, "Market Frictions and the Preferred Habitat Theory of the Term Structure of Interest Rates," working paper, University of Maryland.

Barberis, N. and A. Shleifer, 2003, "Style Investing," Journal of Financial Economics, 68, 161-199.

Buraschi, A. and A. Jiltsov, 2007, "Habit Formation and Macroeconomic Models of the Term Structure of Interest Rates," Journal of Finance, 62, 3009-3063.

Campbell, J. and R. Shiller, 1991, "Yield Spreads and Interest Rate Movements: A Bird's Eye View," Review of Economic Studies, 58, 495-514.

Chan, K., G. Karolyi, F. Longstaff, and A. Sanders, 1992, "An Empirical Comparison of Alternative Models of the Short-Term Interest Rate," Journal of Finance, 47, 1209-1227.

Cheridito, P. and X. Gabaix, 2008, "Regularity Conditions to Ensure the Existence of LinearityGenerating Processes," working paper, Princeton University.

Cochrane, J., 2008, "Comments on 'Bond Supply and Excess Bond Returns' by Robin Greenwood and Dimitri Vayanos," working paper, University of Chicago.

Cochrane, J. and M. Piazzesi, 2005, "Bond Risk Premia," American Economic Review, 95, 138-160.

Cox, J., J. Ingersoll, and S. Ross, 1985, "A Theory of the Term Structure of Interest Rates," Econometrica, 53, 385-408.

Culbertson, J., 1957, "The Term Structure of Interest Rates," Quarterly Journal of Economics, $71,485-517$.

Dai, Q. and K. Singleton, 2002, "Expectations Puzzles, Time-Varying Risk Premia, and Affine Models of the Term Structure," Journal of Financial Economics, 63, 415-441.

Duffee, G., 2002, "Term Premia and Interest Rate Forecasts in Affine Models," Journal of Finance, $57,405-443$.

Fama, E. and R. Bliss, 1987, "The Information in Long-Maturity Forward Rates," American Economic Review, 77, 680-692.

Gabaix, X., 2009, "Variable Rare Disasters: An Exactly Solved Framework for Ten Puzzles in Macro-Finance," working paper, New York University. 
Gabaix, X., 2009, "Linearity-Generating Processes: A Modelling Tool Yielding Closed Forms for Asset Prices," working paper, New York University.

Gabaix, X., A. Krishnamurthy, and O. Vigneron, 2007, "Limits of Arbitrage: Theory and Evidence from the Mortgage Backed Securities Market," Journal of Finance, 62, 557-596.

Garbade, K. and M. Rutherford, 2007, "Buybacks in Treasury Cash and Debt Management," Staff Report 304, Federal Reserve Bank of New York.

Garleanu, N., L. Pedersen, and A. Poteshman, 2009, "Demand-Based Option Pricing," Review of Financial Studies, forthcoming.

Greenwood, R., 2005, "Short- and Long-term Demand Curves for Stocks: Theory and Evidence on the Dynamics of Arbitrage," Journal of Financial Economics, 75, 607-649.

Greenwood, R., S. Hanson, and J. Stein, 2009, "A Gap-Filling Theory of Corporate Debt Maturity Choice," Journal of Finance, forthcoming.

Greenwood, R. and D. Vayanos, 2009a, "Bond Supply and Excess Bond Returns," working paper, Harvard University.

Greenwood, R. and D. Vayanos, 2009b, "Price Pressure in the Bond Market," working paper, Harvard University.

Gromb, D. and D. Vayanos, 2002, "Equilibrium and Welfare in Markets with Financially Constrained Arbitrageurs," Journal of Financial Economics, 66, 361-407.

Gromb, D. and D. Vayanos, 2009, "Financially Constrained Arbitrage and Cross-Market Contagion," working paper, INSEAD.

Guibaud, S., Y. Nosbusch, and D. Vayanos, 2009, "Preferred Habitat and the Optimal Maturity Structure of Government Debt," working paper, London School of Economics.

Hau, H., 2009, "Global versus Local Asset Pricing: Evidence from Arbitrage of the MSCI Index Change," working paper, INSEAD.

Islam, M., 2007, "The State that I am in...," Barclays Capital, Equity Gilt Study 2007, 52-63.

Krishnamurthy, A. and A. Vissing-Jorgensen, 2008, "The Aggregate Demand for Treasury Debt," working paper, Northwestern University.

Kyle, A., and W. Xiong, 2001, "Contagion as a Wealth Effect of Financial Intermediaries," Journal of Finance 56, 1401-1440. 
Litterman, R. and J. Scheinkman, 1991, "Common Factors Affecting Bond Returns," Journal of Fixed Income, 1, 54-61.

Modigliani, F. and R. Sutch, 1966, "Innovations in Interest-Rate Policy," American Economic Review, 56, 178-197.

Pavlova, A. and R. Rigobon, 2008, "The Role of Portfolio Constraints in the International Propagation of Shocks," Review of Economic Studies, 75, 1215-1256.

Pradhan, A., 2009, "Forward Steepeners Still Offer Value," Barclays Capital, Global Rates Strategy, 5 June, 2-6.

Telmer, C. and S. Zin, 1996, "The Yield Curve: Terms of Endearment or Terms of Endowment?," working paper, Carnegie-Mellon University.

Tzucker, R. and M. Islam, 2005, "A Pension Reform Primer," Barclays Capital, Fixed Income Rates Strategy.

Vasicek, O., 1977, "An Equilibrium Characterization of the Term Structure," Journal of Financial Economics, 5, 177-188.

Wachter, J., 2006, "A Consumption-Based Model of the Term Structure of Interest Rates," Journal of Financial Economics, 79, 365-399.

Xiong, W. and H. Yan, 2009, "Heterogeneous Expectations and Bond Markets," Review of Financial Studies, forthcoming. 